\title{
Reliable Decentralized Stabilization of Feedforward and Feedback Interconnected Systems
}

\author{
Konur A. Ünyelioğlu, Member, IEEE, and A. Bülent Özgüler
}

\begin{abstract}
Synthesis of decentralized controllers that are reliable in the presence of breakdowns in the interconnections are considered for plants obtained via feedforward and feedback type interconnection schemes. The main objective is to determine the precise conditions on the plant which assure such reliable synthesis. It is shown that the central property for reliable decentralized stabilization is always an appropriate parity interlacing property among the poles of the interconnection subplants and certain blocking zeros of the overall plant. The conditions under which the stabilization of a two-channel plant is affected by the stabilization of its diagonal subplants are also obtained. The results also find application in multiple controller reliable synthesis problems. The discussion is restricted to twochannel plants.
\end{abstract}

\section{INTRODUCTION AND PRELIMINARIES}

A $\mathrm{N}$ important objective in the design of feedback control systems is to assure reliable performance in the face of deviations of the closed-loop parameters from their nominal values. There are many alternative ways of modeling the changes in the parameters. These may roughly be classified as continuous changes such as in robust control or discrete (or on-off) changes such as in the simultaneous stabilization of finitely many plants. Reliable stabilization in the face of "breakdowns" of certain subplants falls into the second category and it has attracted considerable attention in the synthesis of controllers for large-scale systems [10], [9], [16], [11], [8], [14], [1], [5]. A further significance of reliable decentralized stabilization is the application it finds in some reliable full-feedback (centralized) stabilization problems [10], [8]. Our objective is to determine the precise conditions for the existence of decentralized stabilizing compensators which are reliable against breakdowns in the plant inter-

$$
T=\left[\begin{array}{c}
\left(I-T_{11} T_{12} T_{22} T_{21}\right)^{-1} T_{11} \\
\left(I-T_{22} T_{21} T_{11} T_{12}\right)^{-1} T_{22} T_{21} T_{11}
\end{array}\right.
$$

connections and (in some cases) in the external feedback paths. We restrict our study to two-channel plants and to

Manuscript received February 6, 1991; revised November 5, 1991. Paper recommended by Associate Editor, J. Hammer. This work was supported in part by the National Science Foundation under Grant INT-9101276 and by the Scientific and Technical Research Council of Turkey (TUBITAK) under Grant TBAG-1016.

The authors are with the Department of Electrical and Electronics Engineering, Bilkent University, 06533, Bilkent, Ankara, Turkey

IEEE Log Number 9201554

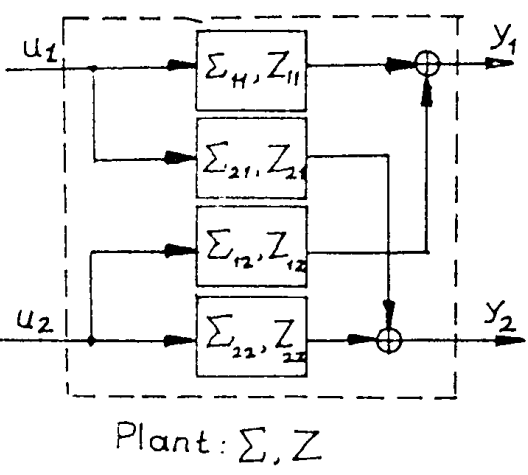

Fig. 1.

stability issues. We consider two different types of interconnection schemes. In Fig. 1, the main subplants $\Sigma_{11}$ and $\Sigma_{22}$ are interconnected via feedforward paths comprising the interconnection subplants $\Sigma_{12}$ and $\Sigma_{12}$. In Fig. 2, on the other hand, the main subplants $\bar{\Sigma}_{11}$ and $\bar{\Sigma}_{22}$ are combined via feedback paths comprising the interconnection subplants $\bar{\Sigma}_{12}$ and $\bar{\Sigma}_{21}$.

If we let $Z_{i j}$ and $T_{i j}$ denote the transfer matrices associated with the subplants $\Sigma_{i j}$ and $\bar{\Sigma}_{i j}$, respectively, for $i, j \in 2$, the overall transfer matrices for the resulting two-channel plants of Figs. 1 and 2 are

$$
Z=\left[\begin{array}{ll}
Z_{11} & Z_{12} \\
Z_{21} & Z_{22}
\end{array}\right]
$$

and

$$
\left.\begin{array}{c}
\left(I-T_{11} T_{12} T_{22} T_{21}\right)^{-1} T_{11} T_{12} T_{22} \\
\left(I-T_{22} T_{21} T_{11} T_{12}\right)^{-1} T_{22}
\end{array}\right]
$$

provided $T_{i j}$ for some $i, j \in 2$ is strictly proper to assure that the interconnection scheme of Fig. 2 is well defined. The same decentralized feedback scheme is considered for the two plants as shown in Fig. 3. To assure that the closed-loop system of Fig. 3 is well defined for both cases, the transfer matrices $Z_{11}, Z_{22}, T_{11}$, and $T_{22}$ are all restricted to be strictly proper.

The main problems we consider in this paper are given below. 


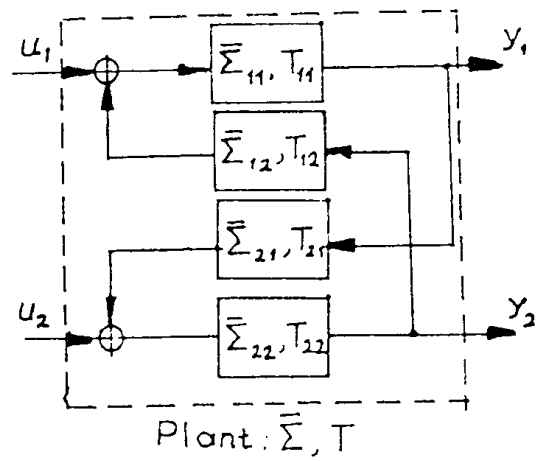

Fig. 2.

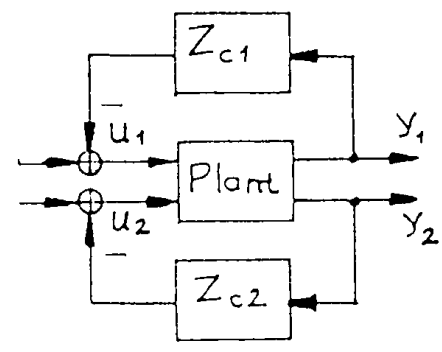

Fig. 3.

P1: Given the feedforward interconnected plant $\Sigma$, determine local compensators $\Sigma_{c 1}$ and $\Sigma_{c 2}$ such that; $i$ ) $\left.\left(\Sigma_{11}, \Sigma_{c 1}\right), i i\right)\left(\Sigma_{22}, \Sigma_{c 2}\right)$, and iii $)\left(\Sigma, \operatorname{diag}\left\{\Sigma_{c 1}, \Sigma_{c 2}\right\}\right)$ are all internally stable.

$P 2$ : Given the feedback interconnected plant $\bar{\Sigma}$, determine local compensators $\Sigma_{c 1}$ and $\Sigma_{c 2}$ such that: $\left.i\right)\left(\bar{\Sigma}_{11}\right.$, $\left.\Sigma_{c 1}\right)$, ii) $\left(\bar{\Sigma}_{22}, \Sigma_{c 2}\right)$, and $\left.i i i\right)\left(\bar{\Sigma}, \operatorname{diag}\left\{\Sigma_{c 1}, \Sigma_{c 2}\right\}\right)$ are all internally stable.

The following table summarizes the type of reliability that follows from the synthesis according to P1 and P2. The reliability properties of P1 listed in the table summarizes the discussion in [8].

An alternative motivation for P1 is obtained by noting that it is the problem of determining conditions under which stabilization of the overall plant is affected by the stabilization of the main subplants $\Sigma_{11}$ and $\Sigma_{22}$. For such applications, it is more suitable to define an input/output version of $\mathrm{P} 1$ which takes the overall two-channel plant as its starting point rather than the subplants.

$P 1_{i / o}$ : Given $Z$ of (1), determine local compensators of transfer matrices $Z_{c 1}$ and $Z_{c 2}$ such that: $\left.\left.i\right)\left(Z_{11}, Z_{c 1}\right), i i\right)$ $\left(Z_{22}, Z_{c 2}\right)$, and $\left.i i i\right)\left(Z, \operatorname{diag}\left\{Z_{c 1}, Z_{c 2}\right)\right\}$ are all internally stable.

The only difference between $\mathrm{P} 1$ and $\mathrm{P} 1_{i / o}$ is that, in $\mathrm{P} 1_{i / o}$, the unstabilizable and undetectable modes (centralized hidden modes) of $\Sigma$ which may be created by the feedforward interconnections are not pertinent to solvability. With this alternative motivation in mind, $\mathbf{P} 1_{i / o}$ has been extensively investigated in the literature and there are many different sufficient solvability conditions. We can cite [6], [18], and the references therein which use quasi block-diagonal dominance methods. Recently, in [1], $\mathrm{P} 1_{i / o}$ has been studied and a solvability condition is given. The main result of [1] is, however, essentially a problem transformation result and is only comparable to our Proposition 2.2. The problem P2 can be viewed as the input-output interconnection counterpart of the state interconnection problem of [11] which in particular considers static interconnection subplants $T_{12}$ and $T_{21}$. The problem has also been considered in [9] with static interconnections $T_{12}, T_{21}$ and local state feedback. We should mention that the sufficient conditions obtained in [9] and [11] apply to general $N$-channel plants.

It turns out that each of the three problems can be transformed either to a simultaneous stabilization problem of two plants, one of which has a parametric representation or to a stabilization problem of a two-channel plant by a stable decentralized compensator. In Section II, we solve $\mathrm{P} 1_{i / o}$ through the first transformation and specialize the obtained result to P1. In Section II, we solve P2 through a transformation of the second type. The main results of the paper are Theorems $2.2,2.3$, and 3.1 which, respectively, yield solutions to $\mathrm{P} 1_{i / o}, \mathrm{P} 1$, and $\mathrm{P} 2$. The solvability condition for $\mathrm{P} 1_{i / o}$ in Theorem 2.2 is in terms of some arbitrary but fixed initial compensators applied at both channels. The main solvability condition for this problem is a parity interlacing condition among the poles and certain blocking zeros of the initial closed-loop system obtained. The solvability conditions of Theorems 2.3 and 3.1 on the other hand are purely in terms of the problem data. The main conditions for both cases is a parity interlacing condition among the poles of the interconnection subplants and certain blocking zeros associated with the overall plant. There is a noteworthy duality between the conditions obtained for $\mathrm{P} 1$ and $\mathrm{P} 2$ which is a consequence of an inherent duality in the problem definitions. In Corollaries $2.1-2.4$ and 3.1, 3.2, the main results are specialized to some cases of independent interest. In Corollary 2.1 and 3.1, the case where the main subplants are stable is examined and it is shown that the main requirement for solvability is that there are an even number of poles between each pair of real positive blocking zeros of the off-diagonal transfer matrices in (1) and (2), respectively. In the case where the interconnection subplants are stable, it is shown in Corollaries 2.2 and 3.2 that, all three problems are solvable. The Corollaries 2.3 and 2.4 concern the application of $\mathrm{P} 1_{i / o}$ to some fullfeedback reliable synthesis problems among which the multiple controller reliable synthesis problem of Šiljak [10] can be mentioned. (Also see [16], [8], [5] and [1].) All these results do provide intuition into the structure of feedforward and feedback interconnected systems in spite of the fact that our consideration is limited to two-channel cases and some general statements can be made. These are listed in the section on conclusions. We also note that although our discussion below is restricted to continuoustime systems, the generalizations to discrete-time systems and to systems of arbitrary stability sets is straightforward by the results of [15]. 
TABLE I

\begin{tabular}{|c|c|c|c|}
\hline & $\begin{array}{l}\text { Type of Failure } \\
\text { Assured Stability }\end{array}$ & $\begin{array}{l}\text { Subplant interconnection } \\
\text { failures modeled as } \\
\text { P1: } Z_{12}=0 \text { and } / \text { or } Z_{21}=0 \\
\text { P2: } T_{12}=0 \text { and } / \text { or } T_{21}=0\end{array}$ & $\begin{array}{l}\text { Feedback interconnection } \\
\text { failures modeled as } \\
Z_{\mathrm{II}}=0 \text { or } Z_{c 2}=0\end{array}$ \\
\hline \multirow[t]{2}{*}{ P1 } & $\begin{array}{l}\text { Stability of the diagonal } \\
\text { subplants }\end{array}$ & $\Sigma_{11}$ and $\Sigma_{22}$ remain stabilized & $\begin{array}{l}\text { The diagonal subplant which } \\
\text { is connected to the functional } \\
\text { compensator remains stabilized }\end{array}$ \\
\hline & $\begin{array}{l}\text { Stability of the overall } \\
\text { closed-loop system }\end{array}$ & $\begin{array}{l}\text { The overall closed-loop plant } \\
\text { remains stabilized provided the } \\
\text { resulting open-loop plant has } \\
\text { no unstable decentralized fixed } \\
\text { modes }\end{array}$ & $\begin{array}{l}\text { The set of the unstable poles } \\
\text { of the overall closed-loop } \\
\text { plant is a subset of the unstable } \\
\text { poles of the open-loop plant }\end{array}$ \\
\hline \multirow[t]{2}{*}{$\mathrm{P} 2$} & $\begin{array}{l}\text { Stability of the diagonal } \\
\text { subplants }\end{array}$ & $\bar{\Sigma}_{11}$ and $\bar{\Sigma}_{22}$ remain stabilized & $\begin{array}{l}\text { The diagonal subplant which } \\
\text { is connected to the functional } \\
\text { compensator remains stabilized }\end{array}$ \\
\hline & $\begin{array}{l}\text { Stability of the overall } \\
\text { closed-loop system }\end{array}$ & $\begin{array}{l}\text { The overall closed-loop plant } \\
\text { remains stabilized provided the } \\
\text { resulting open-loop plant has } \\
\text { no unstable decentralized fixed } \\
\text { modes }\end{array}$ & Not assured \\
\hline
\end{tabular}

We now briefly mention some additional notation to the ones already used. By $\mathscr{C}$ and $\mathscr{R}$, we denote the fields of complex and real numbers, respectively. The symbol $\mathscr{C}_{+e}$ denotes the points on the extended unstable region of the complex plane including infinity and $\mathscr{R}_{+e}:=\mathscr{R} \cap$ $\mathscr{C}_{+e}$. Thus, $\mathscr{R}_{+e}$ is the set of real positive numbers including infinity. The set of transfer matrices are denoted by $\boldsymbol{P}$ and the set of stable transfer matrices by $S$. A complex number $z_{0}$ is a blocking zero of $Z \in P^{p \times r}$ if $Z\left(z_{0}\right)=0$. If $Z$ is stable, then the unstable blocking zeros are the unstable zeros of the smallest invariant factor (SIF) of $Z$ over $S$. Let $\mathscr{S}_{1}$ and $\mathscr{S}_{2}$ be two finite collections of numbers in $\mathscr{R}_{+e}$, in which some numbers may occur more than once. If $\mathscr{S}_{1}$ and $\mathscr{S}_{2}$ are disjoint, then we say that the ordered pair $\left(\mathscr{S}_{1}, \mathscr{S}_{2}\right)$ has parity interlacing property if there are an even number of elements from $\mathscr{S}_{1}$ between each pair of elements from $\mathscr{S}_{2}$. The terminology is borrowed from [19] in which $\mathscr{S}_{1}$ and $\mathscr{S}_{2}$ are, respectively, the poles (with multiplicity) and the blocking zeros of a transfer matrix. Note that, if $\mathscr{P}_{1}$ is the set of $\mathscr{R}_{+e}$ zeros with multiplicity of $a \in S$, then $a(z)$ takes the same sign at all elements $z \in \mathscr{S}_{2}$ if and only if $\left(\mathscr{S}_{1}, \mathscr{S}_{2}\right)$ has the parity interlacing property. If $q$ is an integer, $\boldsymbol{q}$ denotes the ordered set $\{1,2, \cdots, q\}$. By $I_{s}$, we denote the identity matrix of size $S$, the subscript is dropped if the size is clear from the context. The determinant of a square matrix $A$ is denoted by $|A|$ and the transpose of a matrix $B$ by $B^{\prime}$. Occasionally, the abbreviation "iff" is used for "if and only if." For all other undefined terminology and notation pertaining to the algebraic and topological structure of the ring $S$ and for matrices over $S$, we refer the reader to [15] and [7].

\section{FEEDFORWARD INTERCONNECTED SySTEMS}

We first consider $\mathrm{P} 1_{i / o}$, a solution to which immediately yields a solution to $\mathrm{P} 1$. The solutions to $\mathrm{P} 1_{i / o}$ and
P1 are obtained by exploiting some recent results in [7]. The main idea is to build the solution upon the characterization result in [7] of all solutions to the single channel canonicity problem with internal stability (SCCPIS). It turns out that $\mathrm{P} 1_{i / o}$ is equivalent to simultaneously stabilizing $Z_{11}$ and any one of the closed-loop transfer matrices attainable by a solution to SCCPIS (see Theorem 2.1 below). The standard result of [15] on simultaneous stabilization and some genericity arguments then yield the main result Theorem 2.2 .

Let the overall open-loop transfer matrix $Z$ be written in a bicoprime fractional representation over $S$ as

$$
Z=\left[\begin{array}{ll}
Z_{11} & Z_{12} \\
Z_{21} & Z_{22}
\end{array}\right]=\left[\begin{array}{l}
P_{1} \\
P_{2}
\end{array}\right] Q_{11}^{-1}\left[\begin{array}{ll}
R_{1} & R_{2}
\end{array}\right]
$$

where $P_{i} \in S^{p_{i} \times q}, R_{i} \in S^{q \times r_{i}}, i \in \mathbf{2}$ and $Q_{11} \in \boldsymbol{S}^{q \times q}$ is nonsingular. It is well known that the decentralized stabilization problem (DSP) [17], [2], [3], [7], [12] is solvable for $Z$, that is for some $\bar{Z}_{c 1} \in \boldsymbol{P}^{r_{1} \times p_{1}}$ and $\bar{Z}_{c 2} \in \boldsymbol{P}^{r_{2} \times p_{2}}(Z$, $\left.\operatorname{diag}\left\{\bar{Z}_{c 1}, \bar{Z}_{c 2}\right\}\right)$ is internally stable, if and only if $\left(P_{1}, Q_{11}\right.$, $\left.R_{2}, 0\right)$ and $\left(P_{2}, Q_{11}, R_{1}, 0\right)$ are complete quadruples, in other words, for all $z \in \mathscr{C}_{+e}$, it holds that

$$
\begin{aligned}
\operatorname{rank}\left[\begin{array}{cc}
Q_{11} & R_{2} \\
-P_{1} & 0
\end{array}\right] \geq q=\operatorname{size}\left(Q_{11}\right) \\
\operatorname{rank}\left[\begin{array}{cc}
Q_{11} & R_{1} \\
-P_{2} & 0
\end{array}\right] \geq q=\operatorname{size}\left(Q_{11}\right) .
\end{aligned}
$$

The completeness conditions are equivalent to the condition that $Z$ has no unstable decentralized fixed modes. Since by the problem definition, the solvability of $\mathrm{P} 1_{i / o}$ requires the solvability of DSP, we assume that the completeness conditions above hold. It is further assumed that $Z$ is strongly connected [2], [7], i.e., both $Z_{12}$ and $Z_{21}$ are nonzero. If $Z$ is not strongly connected, the plant transfer 
matrix is upper and/or lower triangular, and any $Z_{c 1}, Z_{c 2}$ such that $\left(Z_{11}, Z_{c 1}\right)$ and $\left(Z_{22}, Z_{c 2}\right)$ are internally stable, also solves $\mathrm{P} 1_{i / 0}$.

We now summarize certain relevant results of [7].

Given (3), let $C=\operatorname{gcrf}\left(P_{2}, Q_{11}\right)$ so that $Q_{11}=Q_{2} C$, and $P_{2}=P C$ for a right-coprime pair $\left(P, Q_{2}\right)$ where "gcrf" is an abbreviation for "a greatest common right factor." Also let $D=\operatorname{gcl} f\left(Q_{2}, R_{2}\right)$ so that $Q_{2}=D Q$ and $R_{2}=D R$ for a left-coprime pair of matrices $(Q, R)$, where "gclf" stands for "a greatest common left factor." Consider the generalized Bezout identities

$\left[\begin{array}{cc}E & F \\ -R_{l} & Q_{l}\end{array}\right]\left[\begin{array}{cc}Q & -F_{r} \\ P & E_{r}\end{array}\right]=I,\left[\begin{array}{cc}Q & R \\ -H_{l} & G_{l}\end{array}\right]\left[\begin{array}{cc}G & -P r \\ H & Q_{r}\end{array}\right]=I$

for some matrices $E, F, E_{r}, F_{r}, Q_{l}, R_{l}, G, H, G_{l}, H_{l}, Q_{r}$, and $P_{r}$ over $S$ and let

$$
P_{c 2}(X):=H F_{r}+Q_{r} X, Q_{c 2}(X):=E_{r}+P G F_{r}-P P_{r} X
$$

for $X \in S^{r_{2} \times p_{2}}$. It follows by the standard Youla-Bongiorno-Jabr-Kučera parameterization that $\left(Z_{22}, Z_{c 2}\right)$ is internally stable if and only if $Z_{c 2}=$ $P_{c 2}(X) Q_{c 2}(X)^{-1}$ for some $X \in S^{r_{2} \times p_{2}}$ (see [15], [7] for details). Also note that for all $X \in S^{r_{2} \times p_{2}},\left(P_{c 2}(X)\right.$, $\left.Q_{c 2}(X)\right)$ is a coprime pair. Applying the compensator $Z_{c 2}=P_{c 2}(X) Q_{c 2}(X)^{-1}$ to the second channel, the closed-loop transfer matrix around the first channel, denoted by $\hat{Z}_{11}(X)$, becomes

$$
\hat{Z}_{11}(X)=\left[\begin{array}{ll}
P_{1} & 0
\end{array}\right]\left[\begin{array}{cc}
D Q C & D R P_{c 2}(X) \\
-P C & Q_{c 2}(X)
\end{array}\right]^{-1}\left[\begin{array}{c}
R_{1} \\
0
\end{array}\right] .
$$

A compensator $Z_{c 2}$ is a solution to SCCPIS if and only if there exists $X$ such that $Z_{c 2}=P_{c 2}(X) Q_{c 2}(X)^{-1}$ and the fractional representation in (6) is bicoprime. In this case, by [7, theorem 32], $\operatorname{diag}\left\{Z_{c 1}, Z_{c 2}\right\}$ solves DSP if and only if $\left(\hat{Z}_{11}(X), Z_{c 1}\right)$ is internally stable. On the other hand, [7, theorem 33] yields a characterization of all solutions to SCCPIS. The crucial feature of this result is that almost all choices for $X$ yields a solution to SCCPIS, provided the original plant is free of unstable decentralized fixed modes. These results of $[7$, theorems $3.2,3.3]$ are summarized in the following proposition in which the precise description of $\mathscr{X}$ is left out as it is not needed for the subsequent considerations.

Proposition 2.1: Let the plant $Z$ have no unstable decentralized fixed modes. Consider $Z_{c 2}=P_{c 2}(X) Q_{c 2}(X)^{-1}$, where $P_{c 2}(X)$ and $Q_{c 2}(X)$ are given by $(5)$.

a) $\operatorname{diag}\left\{Z_{c 1}, Z_{c 2}\right\}$ solves DSP for some $Z_{c 1}$ if and only if (6) is a bicoprime fraction and $\left(\hat{Z}_{11}(X), Z_{c 1}\right)$ is internally stable.

b) There exists an open and dense subset $\mathscr{X} \subseteq S^{r_{2} \times p_{2}}$ such that (6) is a bicoprime fraction if and only if $X \in \mathscr{X}$.

We are now able to formulate $\mathrm{P}_{i / 0}$ as a simultaneous stabilization problem.
Theorem 2.1: Let $Z$ have no unstable decentralized fixed modes. The problem $\mathrm{P} 1_{i / o}$ is solvable if and only if there exists $X \in \mathscr{Z}$ such that $Z_{11}$ and $\hat{Z}_{11}(X)$ are simultaneously stabilizable.

Proof: [Only If] If $Z_{c 1}, Z_{c 2}$ solve $\mathrm{P} 1_{i / o}$, by ii) of $\mathrm{P} 1_{i / o},\left(Z_{22}, Z_{c 2}\right)$ is internally stable, i.e., $Z_{c 2}=$ $P_{c 2}(X) Q_{c 2}(X)^{-1}$ for some $X \in S^{r_{2} \times p_{2}}$. By Proposition 2.1 , the solvability of DSP implies that (6) is bicoprime and hence $X \in \mathscr{X}$. By i) and iii), respectively, $\left(Z_{11}, Z_{c 2}\right)$ and $\left(\hat{Z}_{11}(X), Z_{c 1}\right)$ are also internally stable. Hence $\hat{Z}_{11}(X)$ and $Z_{11}$ are simultaneously stabilizable. [If] We have $\left(Z_{22}\right.$, $Z_{c 2}$ ) is internally stable by the choice $Z_{c 2}=$ $P_{c 2}(X) Q_{c 2}(X)^{-1}$ where $X \in \mathscr{X}$. So, ii) of the problem statement is satisfied. Let $Z_{c 1}$ be such that $\left(Z_{11}, Z_{c 1}\right)$ and $\left(\hat{Z}_{11}(X), Z_{c 1}\right)$ are internally stable. In this case $\left.\mathrm{i}\right)$ is also satisfied. To show $\left(Z, \operatorname{diag}\left\{Z_{c 1}, Z_{c 2}\right\}\right)$ is internally stable, it is enough to show (6) is bicoprime, since then, the result follows by part a) of Proposition 2.1. But this follows by part b) of Proposition 2.1 and the fact that $X \in \mathscr{X}$.

We now make use of [15, theorem 5.4.2] to reduce our simultaneous stabilization problem to a parity interlacing condition. For this purpose, let

$$
Z_{11}=D_{l}^{-1} N_{l}=N_{r} D_{r}^{-1}
$$

be coprime fractional representations of $Z_{11}$ so that there exist matrices $\bar{\Psi}_{1}, \bar{\Psi}_{2}, \Psi_{1}, \Psi_{2}$ satisfying

$$
\left[\begin{array}{cc}
\Psi_{1} & \Psi_{2} \\
-N_{l} & D_{l}
\end{array}\right]\left[\begin{array}{cc}
D_{r} & -\bar{\Psi}_{2} \\
N_{r} & \bar{\Psi}_{1}
\end{array}\right]=I
$$

Also let

$$
D^{-1} R_{1}=\bar{R}_{1} \bar{D}^{-1}
$$

for some right-coprime pair $\left(\bar{R}_{1}, \bar{D}\right)$. Consider the matrices

$$
\begin{gathered}
\Theta:=\left[\begin{array}{cc}
C & \left(E+G F_{r} R_{l}\right) \bar{R}_{1} \\
-\Psi_{2} P_{1} & \Psi_{1} \bar{D}
\end{array}\right], \Gamma_{1}:=\left[\begin{array}{c}
0 \\
I_{p_{1}}
\end{array}\right] \\
\Lambda_{1}:=\left[\begin{array}{ll}
D_{l} P_{1} & N_{l} \bar{D}
\end{array}\right] \\
\Gamma_{2}:=\left[\begin{array}{c}
P_{r} \\
0
\end{array}\right], \Lambda_{2}:=\left[\begin{array}{ll}
0 & R_{l} \bar{R}_{1}
\end{array}\right] .
\end{gathered}
$$

Let us also define

$$
\Pi:=\left[\begin{array}{cc}
C & \left(E+G F_{r} R_{l}\right) \bar{R}_{1} \\
D_{l} P_{1} & N_{l} \bar{D}
\end{array}\right], \Gamma_{0}:=\left[\begin{array}{c}
P_{r} \\
0
\end{array}\right]
$$

where the matrices $\Gamma_{2}$ and $\Gamma_{0}$ are the same except for the size of their zero blocks, i.e., $\Gamma_{2}$ is of size $\left(q+r_{1}\right) \times r_{2}$ whereas $\Gamma_{0}$ is of size $\left(q+p_{1}\right) \times r_{2}$.

Proposition 2.2: Let $X \in \mathscr{X}$. Then, $Z_{11}$ and $\hat{Z}_{11}(X)$ are simultaneously stabilizable if and only if $\left|\Theta-\Gamma_{0} X \Lambda_{2}\right|$ takes the same sign at $z \in \mathscr{S}(X)$, where

$$
\mathscr{S}(X):=\left\{z \in \mathscr{R}_{+e}: \operatorname{rank}\left[\Pi-\Gamma_{2} X \Lambda_{2}\right](z)=q\right\} .
$$


Proof: Let $X \in \mathscr{X}$ be fixed. Note from (4)-(6) that

$$
\hat{Z}_{11}(X)=P_{1} C^{-1}\left[\left(E+G F_{r} R_{l}\right) \bar{R}_{1}-P_{r} X R_{l} \bar{R}_{1}\right] \bar{D}^{-1} \text {. }
$$

Let

$$
C^{-1}\left[\left(E+G F_{r} R_{l}\right) \bar{R}_{1}-P_{r} X R_{l} \bar{R}_{1}\right]=L(X) K(X)^{-1}
$$

for some right-coprime $(L(X), K(X))$. Since $X \in \mathscr{X}$, by Proposition 2.1-b, it follows that $\left[P_{1} L(X)\right][\bar{D} K(X)]^{-1}$ is a right-coprime fraction of $\hat{Z}_{11}(X)$. Employing [15, theorem 5.4.2], the pair of plants $Z_{11}, \hat{Z}_{11}(X)$ are simultaneously stabilizable if and only if $|\Theta(X)|$ takes the same sign at $\mathscr{R}_{+e}$-blocking zeros of $\Psi(X)$, where

$$
\left[\begin{array}{c}
\Theta(X) \\
\Psi(X)
\end{array}\right]=\left[\begin{array}{cc}
\Psi_{1} & \Psi_{2} \\
-N_{I} & D_{l}
\end{array}\right]\left[\begin{array}{l}
\bar{D} K(X) \\
P_{1} L(X)
\end{array}\right] .
$$

It remains to show that $|\Theta(X)|=\left|\Theta-\Gamma_{2} X \Lambda_{2}\right|$ and that $\mathscr{R}_{+}$-blocking zeros of $\Psi(X)$ are given by the set $\mathscr{S}(X)$. In fact

$$
\begin{aligned}
|\Theta(X)| & =\left|\left[\begin{array}{cc}
I_{q} & L(X) \\
0 & \Theta(X)
\end{array}\right]\right|=\left|\left[\begin{array}{cc}
I_{q} & L(X) \\
-\Psi_{2} P_{1} & \Psi_{1} \bar{D} K(X)
\end{array}\right]\right| \\
& =\left|\left[\begin{array}{cc}
C & E \bar{R}_{1}+\left(G F_{r}-P_{r} X\right) R_{l} \bar{R}_{1} \\
-\Psi_{2} P_{1} & \Psi_{1} \bar{D}
\end{array}\right]\right| \\
& =\left|\Theta-\Gamma_{2} X \Lambda_{2}\right| .
\end{aligned}
$$

On the other hand, observe that the set of $\mathscr{R}_{+e}$-blocking zeros of $\Psi(X)$ are the same as $\left\{z \in \mathscr{R}+e^{\mid \text {rank }}\right.$ $\left.\left[\begin{array}{cc}I_{q} & 0 \\ 0 & \Psi(X)\end{array}\right](z)=q\right\}$. By simple manipulations and utilizing (4), it is not difficult to see that

$$
\begin{aligned}
& \operatorname{rank}\left[\begin{array}{cc}
I_{q} & 0 \\
0 & D_{l} P_{1} L(X)-N_{l} \bar{D} K(X)
\end{array}\right]=q \text { iff } \\
& \operatorname{rank}\left[\begin{array}{cc}
C & E \bar{R}_{1}+\left(G F_{r}-P_{r} X\right) R_{l} \bar{R}_{1} \\
D_{l} P_{1} & N_{l} \bar{D}
\end{array}\right]=q
\end{aligned}
$$

for all $z \in \mathscr{C}_{+e}$. The antecedent of the above proposition is precisely rank $\left[\Pi-\Gamma_{0} X \Lambda_{2}\right]=q$ and the result follows.

Remark 2.1: The introduction of the matrices in (8) can be further motivated by transforming $\mathrm{P}_{i / o}$ to an equivalent problem of decentralized strong stabilization. Note that any compensator which internally stabilizes $Z_{11}$ is of the form $Z_{c 1}=\left(\Psi_{1}-Y N_{l}\right)^{-1}\left(\Psi_{2}+Y D_{l}\right)$ for some $Y \in$ $S^{r_{1} \times p_{1}}$. Applying $Z_{c 1}$ to $\hat{Z}_{11}(X)$, one obtains an autonomous plant of the denominator matrix

$$
\begin{aligned}
& \hat{\Phi}(X, Y) \\
& :=\left[\begin{array}{ccc}
D Q C & R_{1} & D R\left(H F_{r}+Q_{r} X\right) \\
-\left(\Psi_{2}+Y D_{l}\right) P_{1} & \Psi_{1}-Y N_{l} & 0 \\
-P C & 0 & E_{r}+P G F_{r}-P P_{r} X
\end{array}\right] .
\end{aligned}
$$

By simple manipulations, it is easy to see that $\mid \hat{\Phi}(X$, $Y)|=| \Theta-\Gamma_{1} Y \Lambda_{1}-\Gamma_{2} X \Lambda_{2} \mid$, where the matrices on the right-hand side are as defined in (8). It follows that the problem $\mathrm{P} 1_{i / o}$ has a solution if and only if there exist $Y \in S^{r_{1} \times p_{1}}$ and $X \in S^{r_{2} \times p_{2}}$ such that $\Theta-\Gamma_{1} Y \Lambda_{1}-$ $\Gamma_{2} X \Lambda_{2}$ is unimodular over $S$. In other words, $\mathrm{P} 1_{i / o}$ is solvable iff there exists a stable decentralized compensator which stabilizes the subsidiary two-channel plant of transfer matrix

$$
\left[\begin{array}{ll}
\hat{Z}_{11} & \hat{Z}_{12} \\
\hat{Z}_{21} & \hat{Z}_{22}
\end{array}\right]=\left[\begin{array}{l}
\Lambda_{1} \\
\Lambda_{2}
\end{array}\right] \Theta^{-1}\left[\begin{array}{ll}
\Gamma_{1} & \Gamma_{2}
\end{array}\right]
$$

The matrices $\left[\begin{array}{ll}\Pi & \Gamma_{0}\end{array}\right]$ and $\left[\begin{array}{ll}\Pi^{\prime} & \Lambda_{2}^{\prime}\end{array}\right]^{\prime}$ that occur in the description of the set $\mathscr{S}$ of Proposition 2.3 below, on the other hand, are (in essence) the system matrices associated with $\left[\begin{array}{ll}\hat{Z}_{11} & \hat{Z}_{12}\end{array}\right]$ and $\left[\hat{Z}_{11}^{\prime} \quad \hat{Z}_{21}^{\prime}\right]^{\prime}$, respectively. $\Delta$

The next step is determining the precise conditions under which an $X \in \mathscr{X}$ exists such that $\left|\Theta-\Gamma_{0} X \Lambda_{2}\right|$ takes the same sign at all $z \in \mathscr{S}$. For this purpose, we first identify the elements of $\mathscr{P}(X)$ which are independent of $X$.

Consider the connectivity condition

$$
\operatorname{rank}\left(Z_{12}\right) \geq 2 \text { or } \operatorname{rank}\left(Z_{21}\right) \geq 2 \text {. }
$$

Proposition 2.3: Let

$$
\begin{aligned}
\mathscr{S} & :=\left\{z \in \mathscr{R}_{+e}: \operatorname{rank}\left[\begin{array}{ll}
\Pi & \Gamma_{0}
\end{array}\right](z)\right. \\
& \left.=q \text { or rank }\left[\begin{array}{c}
\Pi \\
\Lambda_{2}
\end{array}\right](z)=q\right\} .
\end{aligned}
$$

i) For all $X \in \mathscr{X}$, the inclusion $\mathscr{S} \subset \mathscr{S}(X)$ holds.

ii) If (10) holds, then there exists an open and dense subset $\overline{\mathscr{X}}$ of $\mathscr{X}$ such that $\mathscr{S}=\mathscr{S}(X)$ for all $X \in \overline{\mathscr{X}}$.

Proof: See the Appendix.

Thus, the elements of the set $\mathscr{S}$ are the $\mathscr{R}_{+e}$ blocking zeros of $\mathscr{S}(X)$ that are independent of $X \in \mathscr{X}$. If rank $Z_{12} \geq 2$ or rank $Z_{21} \geq 2$, then the set of $\mathscr{R}_{+e}$-blocking zeros of $\mathscr{S}(X)$ is precisely $\mathscr{S}$ for almost all elements of $\mathscr{X}$. In other words, when (10) holds the following is true: If $X_{0} \in \mathscr{X}$ is such that $\mathscr{S}\left(X_{0}\right)=\mathscr{S}$, then the equality still holds under sufficiently small perturbations on $X_{0}$. On the other hand, if for some $X_{0} \in \boldsymbol{S}^{r_{2} \times p_{2}}, \mathscr{S}\left(X_{0}\right)$ contains some zeros different than those in $\mathscr{S}$, there exists an arbitrarily small perturbation on $X_{0}$ such that these zeros are eliminated, so that $\mathscr{S}\left(X_{0 p}\right)=\mathscr{S}$, where $X_{0 p} \in \mathscr{X}$ is the suitable perturbation of $X_{0}$.

Consider the set

$$
\mathscr{T}:=\left\{z \in \mathscr{R}_{+e}:\left(\Lambda_{2} \Theta^{-1} \Gamma_{2}\right)(z)=0\right\}
$$

which is the set of real positive blocking zeros of the transfer matrix $\hat{Z}_{22}$ of Remark 2.1. We can now give the main result of this section.

Theorem 2.2: Suppose (10) holds. Then, $\mathrm{P} 1_{i / o}$ is solvable if and only if $Z$ has no unstable decentralized fixed modes and $|\Theta|$ takes the same sign at $z \in \mathscr{S} \cap \mathscr{T}$, where $\Theta, \mathscr{S}$, and $\mathscr{T}$ are as defined by (8), (11), and (12). 
Proof: Let $\Omega_{l}:=\operatorname{gclf}\left(\Theta, \Gamma_{2}\right)$ so that $\Theta=\Omega_{l}, \bar{\Theta}, \Gamma_{2}=$ $\Omega_{l} \hat{\Gamma}$ for some left coprime pair of matrices $(\bar{\Theta}, \hat{\Gamma})$. Also let $\Omega_{r}:=\operatorname{gcrf}\left(\bar{\Theta}, \Lambda_{2}\right)$ so that $\bar{\Theta}=\hat{\Theta} \Omega_{r}, \Lambda_{2}=\hat{\Lambda} \Omega_{r}$ for some right coprime pair $\hat{\Theta}, \hat{\Lambda}$. Then, $\Lambda_{2} \Theta^{-1} \Gamma_{2}=\hat{\Lambda} \hat{\Theta}^{-1} \hat{\Gamma}$, where the latter is a bicoprime fractional representation. Moreover

$$
\left|\Theta-\Gamma_{2} X \Lambda_{2}\right|=\left|\Omega_{l}\right||\hat{\Theta}-\hat{\Gamma} X \hat{\Lambda}|\left|\Omega_{r}\right|
$$

and if $A^{-1} B$ is a left-coprime fractional representation of $\Lambda_{2} \Theta^{-1} \Gamma_{2}$, then $\left|\Theta-\Gamma_{2} X \Lambda_{2}\right|$ and $\left|\Omega_{l}\right||A-B X|\left|\Omega_{r}\right|$ are associates, i.e., they are equal up to a multiplication by a unit in $S$. Since this is true for all $X$ and for $X=0$ in particular, $|\Theta|$ and $\left|\Omega_{l}\right|\left|\Omega_{r}\right||A|$ as well as $\left|\Omega_{l}\right||\hat{\Theta}|\left|\Omega_{r}\right|$ are associates. Finally, note that $z \in \mathscr{T}$ if and only if $B(z)=0$.

[Only If] By Proposition 2.2, if $\mathrm{P} 1_{i / o}$ has a solution, then there exists $X \in \mathscr{X}$ such that $\left|\Theta-\Gamma_{2} X \Lambda_{2}\right|$ takes the same sign at all $z \in \mathscr{S}(X)$. By Proposition 2.3-i), for all $X \in \mathscr{X}$ one has $\mathscr{S} \subset \mathscr{S}(X)$. Hence, $\left|\Theta-\Gamma_{2} X \Lambda_{2}\right|$ has the same sign at all elements of $\mathscr{S}$. On the other hand, at the elements in $\mathscr{T},\left|\Theta-\Gamma_{2} X \Lambda_{2}\right|$ has constant sign if and only if the sign of $|\Theta|$ is constant since $\mathscr{T}=\left\{z \in \mathscr{R}_{+e}: B=0\right\}$. Therefore, the solvability of $\mathrm{P} 1_{i / o}$ implies that the sign of $|\Theta|$ is the same for all $z \in \mathscr{S} \cap \mathscr{T}$.

[If] Say $\left|\Omega_{l}\right|\left|\Omega_{r}\right||A|>0$ for all $z \in \mathscr{S} \cap \mathscr{T}$. If the reverse inequality holds, the proof below can be modified accordingly. Let $\overline{\mathscr{S}}$ be the complement of $\mathscr{S} \cap \mathscr{T}$ in $\mathscr{S}$, i.e., $\overline{\mathscr{S}}:=\mathscr{S}-\mathscr{S} \cap \mathscr{T}$. Note that, if we denote $\sigma:=$ sifB, then $\sigma(z) \neq 0$ for all $z \in \overline{\mathscr{S}}$ since $\overline{\mathscr{S}}$ does not contain any elements from $\mathscr{T}$. Let $\overline{\mathscr{S}}$ consist of $\left\{z_{i}: i \in \boldsymbol{\nu}\right\}$. We will now construct $X \in \mathscr{X}$ such that $\left|\Theta-\Gamma_{2} X \Lambda_{2}\right|$ has the same sign at all elements of $\mathscr{S}$. For each $z_{i} \in \overline{\mathscr{S}}$, there exists $d_{i} \in \mathscr{R}$ such that $\left|\Omega_{l}\right|\left|\Omega_{r}\right|\left(z_{i}\right)\left(|A|\left(z_{i}\right)-\sigma\left(z_{i}\right) d_{i}\right)>$ 0 . One can construct $x \in S$ by any interpolation technique such that $x\left(z_{i}\right)=d_{i}, i \in v$. Hence $\left|\Omega_{l}\right|\left|\Omega_{r}\right|(|A|-$ $\sigma x)>0$ whenever $z \in \overline{\mathscr{S}}$. By [15, theorem 4.4.2], there exists $X \in S^{r_{2} \times p_{2}}$ such that $|A|-\sigma x=|A-B X|$. That is, there exist $X$ such that $\left|\Omega_{l}\right|\left|\Omega_{r}\right||A-B X|>0$, whenever $z \in \overline{\mathscr{S}}$. Noting that $\left|\Omega_{l}\right|\left|\Omega_{r}\right||A-B X|$ and $\mid \Theta-$ $\Gamma_{2} X \Lambda_{2} \mid$ are associates, the latter takes the same sign at $z \in \mathscr{S}$ with this construction of $X$. Since $\mathscr{X}$ is open and dense in $S^{r_{2} \times p_{2}}, X$ can be assumed to be contained in $\mathscr{X}$, possibly with an arbitrarily small perturbation on $X$, which does not change the sign of $\left|\Theta-\Gamma_{2} X \Lambda_{2}\right|$ at $z \in \mathscr{S}$. By Proposition 2.3-ii) we can also assure that the $\mathscr{R}+e^{\text {-block- }}$ ing zeros of $\Pi-\Gamma_{0} X \Lambda_{2}$ are precisely the elements of $\mathscr{S}$. Otherwise, there exists an arbitrarily small perturbation $X_{p}$ of $X$ such that this property holds. In addition, the perturbation can be restricted to be sufficiently small such that $\left|\Theta-\Gamma_{2} X_{p} \Lambda_{2}\right|$ still has the same sign at all $z \in \mathscr{S}$. The result now follows by Proposition 2.2 and Theorem 2.1 .

We now use the result of Theorem 2.2 to get a solution to P1.

Let

$$
Z_{i j}=D_{i j}^{-1} N_{i j}=\bar{N}_{i j} \bar{D}_{i j}^{-1}
$$

be some left- and right-coprime fractional representations for $i, j \in \mathbf{2}$. It is easy to verify that the overall transfer matrix $Z$ has the following fractional representation in terms of the left-coprime fractional representations for the subplants $Z_{i j}$ above

$$
\begin{array}{r}
Z=\left[\begin{array}{llll}
I & I & 0 & 0 \\
0 & 0 & I & I
\end{array}\right]\left[\begin{array}{cccc}
D_{11} & 0 & 0 & 0 \\
0 & D_{12} & 0 & 0 \\
0 & 0 & D_{21} & 0 \\
0 & 0 & 0 & D_{22}
\end{array}\right]^{-1} \\
\\
{\left[\begin{array}{cc}
N_{11} & 0 \\
0 & N_{12} \\
N_{21} & 0 \\
0 & N_{22}
\end{array}\right] .}
\end{array}
$$

Let

$$
\begin{gathered}
R_{1}:=\left[\begin{array}{c}
N_{11} \\
0 \\
N_{21} \\
0
\end{array}\right], R_{2}:=\left[\begin{array}{c}
0 \\
N_{12} \\
0 \\
N_{22}
\end{array}\right] \\
P_{1}:=\left[\begin{array}{llll}
I & I & 0 & 0
\end{array}\right], P_{2}:=\left[\begin{array}{llll}
0 & 0 & I & I
\end{array}\right]
\end{gathered}
$$

and $Q_{11}:=\operatorname{diag}\left(D_{11}, D_{12}, D_{21}, D_{22}\right\}$. The decentralized stabilization problem for $\Sigma$ is solvable if and only if the fractional representation is bicoprime, i.e., $\left(Q_{11}, R_{1}, R_{2}\right)$ is left and $\left(P_{1}, P_{2}, Q_{11}\right)$ is right coprime, and the quadruples $\left(P_{1}, Q_{11}, R_{2}, 0\right)$ and $\left(P_{2}, Q_{11}, R_{1}, 0\right)$ are both complete. It is easy to see that the fraction in (13) is bicoprime if and only if

$$
\left(D_{21}, D_{22}\right),\left(D_{12}, D_{11}\right) \text { are right-coprime pairs }
$$

and

$$
\left(\bar{D}_{21}, \bar{D}_{11}\right),\left(\bar{D}_{12}, \bar{D}_{22}\right) \text { are left-coprime pairs. }
$$

Let $\left(\tilde{D}_{11}, \tilde{D}_{21}\right)$ and $\left(\tilde{D}_{22}, \tilde{D}_{12}\right)$ be some left-coprime pairs of matrices satisfying

$$
\bar{D}_{21}^{-1} \bar{D}_{11}=\bar{D}_{11} \tilde{D}_{21}^{-1}, \bar{D}_{12}^{-1} \bar{D}_{22}=\tilde{D}_{22} \tilde{D}_{12}^{-1}
$$

The completeness conditions turn out to be equivalent to the rank inequalities

$$
\begin{aligned}
& \operatorname{rank}\left[\begin{array}{ccc}
D_{21} & N_{21} \bar{D}_{11} & 0 \\
D_{22} & 0 & 0 \\
0 & 0 & D_{12}
\end{array}\right] \geq p_{1}+p_{2} \text { and } \\
& \operatorname{rank}\left[\begin{array}{ccc}
D_{12} & N_{12} \bar{D}_{22} & 0 \\
D_{11} & 0 & 0 \\
0 & 0 & D_{21}
\end{array}\right] \geq p_{1}+p_{2}
\end{aligned}
$$

holding for all $z \in \mathscr{C}_{+e}$. The coprimeness conditions (14), (15) and the rank conditions in (16) are thus necessary 
conditions for the solvability of P1. Let the matrices $G_{i}$, $H_{i}, \bar{G}_{i}, \bar{H}_{i}$ satisfy

$$
\left[\begin{array}{cc}
D_{i i} & N_{i i} \\
-\bar{H}_{i} & \bar{G}_{i}
\end{array}\right]\left[\begin{array}{cc}
G_{i} & -\bar{N}_{i i} \\
H_{i} & \bar{D}_{i i}
\end{array}\right]=I, \quad i \in \mathbf{2}
$$

where by left coprimeness of $\left(D_{11}, N_{11} \bar{D}_{21}\right)$ and of $\left(D_{22}\right.$, $N_{22} \bar{D}_{12}$ ) one can in particular choose $H_{1}=\bar{D}_{21} \hat{H}_{1}$ and $H_{2}=\bar{D}_{12} \hat{H}_{2}$, for some $\hat{H}_{1}$ and $\hat{H}_{2}$. If $\Sigma$ has no unstable centralized fixed modes, then the problem P1 is solvable if and only if $\mathrm{P} 1_{i / o}$ is solvable for the plant (13) and we can make use of Theorem 2.2 with $Q_{11}, P_{i}, R_{i}, i \in \mathbf{2}$ as defined above. Note that with

$$
\begin{gathered}
E:=\left[\begin{array}{cccc}
I & 0 & 0 & 0 \\
0 & I+D_{22} & I & 0 \\
0 & -I & 0 & 0 \\
0 & 0 & 0 & I
\end{array}\right], F_{r}:=\left[\begin{array}{c}
0 \\
-I \\
I \\
0
\end{array}\right], \\
G:=\left[\begin{array}{cccc}
I & 0 & 0 & 0 \\
0 & I & 0 & 0 \\
0 & 0 & G_{2} & 0 \\
0 & 0 & -N_{12} H_{2} & I
\end{array}\right] \\
R_{l}=\left[\begin{array}{llll}
0 & D_{22} & I & 0
\end{array}\right]
\end{gathered}
$$

(4) is satisfied. With these choices, the matrix $\Theta$ is such that

$$
\begin{aligned}
|\Theta| & =\left|\left[\begin{array}{cc}
D_{12} & -N_{12} H_{2} D_{22} \\
N_{21} H_{1} D_{11} & D_{21}
\end{array}\right]\right| \\
& =\left|D_{12}\right|\left|D_{21}\right|\left|I-\bar{N}_{21} \hat{H}_{1} D_{11} \bar{N}_{12} \hat{H}_{2} D_{22}\right| \\
& =\left|D_{12}\right|\left|D_{21}\right|\left|I-\bar{N}_{21} \tilde{D}_{11} \tilde{H}_{1} \bar{N}_{12} \tilde{D}_{22} \tilde{H}_{2}\right| .
\end{aligned}
$$

The set $\mathscr{S}$ is easily shown to simplify to

$$
\begin{aligned}
\mathscr{S}=\left\{z \in \mathscr{R}_{+e} \mid \operatorname{rank}\left[\begin{array}{cc}
D_{12} & N_{12} \bar{D}_{22} \\
D_{11} & 0
\end{array}\right](z)=p_{1}\right. \text { or } \\
\left.\operatorname{rank}\left[\begin{array}{cc}
D_{21} & N_{21} \bar{D}_{11} \\
D_{22} & 0
\end{array}\right](z)=p_{2}\right\}
\end{aligned}
$$

on taking $D_{l}=D_{11}, N_{l}=N_{11}$. By the coprimeness conditions (14), (15), the set $S$ has an even simpler description given by

$$
\begin{aligned}
\mathscr{S}= & \left\{\mathscr{R}_{+e} \text {-blocking zeros of } D_{11} \bar{N}_{12} \tilde{D}_{22}\right\} \\
& \cup\left\{\mathscr{R}_{+e} \text {-blocking zeros of } D_{22} \bar{N}_{21} \tilde{D}_{11}\right\} \\
= & \left\{\mathscr{R}_{+e} \text {-blocking zeros of } D_{11} Z_{12} \bar{D}_{22}\right\} \\
& \cup\left\{\mathscr{R}_{+e} \text {-blocking zeros of } D_{22} Z_{21} \bar{D}_{11}\right\} .
\end{aligned}
$$

On the other hand, the set $\mathscr{T}$ becomes

$$
\begin{aligned}
\mathscr{T}= & \left\{\mathscr{R}_{+e} \text {-blocking zeros of }\left[\begin{array}{ll}
0 & D_{22}
\end{array}\right]\right. \\
& \left.\cdot\left[\begin{array}{cc}
D_{12} & -N_{12} H_{2} D_{22} \\
N_{21} H_{1} D_{11} & D_{21}
\end{array}\right]^{-1}\left[\begin{array}{c}
N_{12} \bar{D}_{22} \\
0
\end{array}\right]\right\} \\
= & \left\{\mathscr{R}_{+e} \text {-blocking zeros of }\left(I-D_{22} \bar{N}_{21} \hat{H}_{1} D_{11} \bar{N}_{12} \hat{H}_{2}\right)^{-1}\right. \\
& \left.\cdot D_{22} \bar{N}_{21} \hat{H}_{1} D_{11} D_{12}^{-1} N_{12} \bar{D}_{22}\right\} .
\end{aligned}
$$

Noting that $\hat{H}_{1} D_{11}=\bar{D}_{21}^{-1} \bar{D}_{11} \bar{H}_{1}$ and $\hat{H}_{2} D_{22}=$ $\bar{D}_{12}^{-1} \bar{D}_{22} \bar{H}_{2}$, we have $\left|I-D_{22} \bar{N}_{21} \hat{H}_{1} D_{11} \bar{N}_{12} \hat{H}_{2}\right|=\mid I-$ $D_{22} Z_{21} \bar{D}_{11} \bar{H}_{1} \bar{N}_{12} \hat{H}_{2}|=| I-\bar{N}_{21} \hat{H}_{1} D_{11} \quad \bar{N}_{12} \hat{H}_{2} D_{22}|=| I$ $-\bar{N}_{21} \hat{H}_{1} D_{11} Z_{12} \bar{D}_{22} \bar{H}_{2}$ l, it follows that $z \in \mathscr{S}$ implies $z \in$ $\mathscr{T}$, i.e., $\mathscr{S} \cap \mathscr{T}=\mathscr{S}$. Moreover, at all zeros in $\mathscr{S}$, it is clear that $|\Theta|=\left|D_{12}\right|\left|D_{21}\right|$. We therefore have the following solution to $\mathbf{P 1}$.

Theorem 2.3: Suppose that either $\operatorname{rank} Z_{12} \geq 2$ or rank $Z_{21} \geq 2$. There exists a solution to $P 1$ if and only if the system $\Sigma$ is free of unstable decentralized fixed modes (equivalently, the coprimeness conditions (14), (15), and the rank conditions in (16) hold) and $\left|D_{12}\right|\left|D_{21}\right|$ takes the same sign at the set

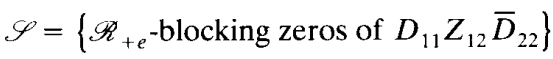

$$
\cup\left\{\mathscr{R}_{+e} \text {-blocking zeros of } D_{22} Z_{21} \bar{D}_{11}\right\} \text {. }
$$

Remark 2.2: i) The connectivity condition (10) is only required in the "if" parts of the proofs of Theorems 2.2 and 2.3. Hence, all plants for which the problems have solutions satisfy the given related parity interlacing conditions. ii) although the solvability condition given by Theorem 2.2 depends on some initial compensators at the first and second channels, the solvability condition of Theorem 2.3 is purely in terms of the problem data. This is mainly due to the fact that, in problem $\mathrm{P} 1$, a suitable choice for the initial compensators that avails more transparent solvability conditions is quite easy to see, whereas in $\mathrm{P} 1_{i / o}$ it is not. This might have been quite natural to expect since $\mathrm{P} 1_{i / o}$ is in fact a more encompassing problem definition than P1. iii) an alternative proof of Theorem 2.3 can also be given in a manner similar to the proof of Theorem 3.1 below. The above proof is however preferable as it exploits and clarifies the connection between $\mathrm{P} 1$ and $\mathrm{P} 1_{i / o}$.

We now consider some other specializations of the result of Theorem 2.3.

Case 1: $Z_{11}$ and $Z_{22}$ are stable transfer matrices. This is the case where the original plants are stable and are combined according to Fig. 1 via possibly unstable interconnections. We observe that by the stability of $Z_{11}$ and $Z_{22}$ the problems $\mathrm{P} 1$ and $\mathrm{P} 1_{i / o}$ are equivalent. We specialize the result of Theorem 2.3 by making the particular choices $D_{11}=I, D_{22}=I, \bar{D}_{11}=I, \bar{D}_{22}=I, H_{1}=0, H_{2}$ 
$=0$. Theorem 2.3 immediately yields the following result provided the connectivity condition (10) holds. With a slight modification in the proof of Theorem 2.2, however, it can be shown that this assumption can be discarded in this special case. We thus have the following extension of a result in [8] which considers two-input, two-output plants.

Corollary 2.1: Let $Z_{11}$ and $Z_{22}$ be stable transfer matrices. Then, $P 1$ is solvable if and only if $\Sigma$ is free of unstable decentralized fixed modes (equivalently

$$
\operatorname{rank}\left[\begin{array}{cc}
D_{12} & 0 \\
0 & N_{21}
\end{array}\right] \geq p_{1}, \operatorname{rank}\left[\begin{array}{cc}
D_{21} & 0 \\
0 & N_{12}
\end{array}\right] \geq p_{2}
$$

for all $\left.z \in \mathscr{C}_{+e}\right)$ and $\left|D_{12}\right|\left|D_{21}\right|$ takes the same sign at the union of the real unstable blocking zeros of $Z_{12}$ and $Z_{21}$.

Case 2: $Z_{12}$ and $Z_{21}$ are stable transfer matrices. This is the case where the original plants $Z_{11}$ and $Z_{22}$ are interconnected according to Fig. 1 with stable interconnection subplants. As in Case 1, there are no unstable hidden modes of $\Sigma$ and hence the problems $\mathrm{P} 1$ and $\mathrm{P} 1_{i / o}$ are equivalent. Moreover, by hypothesis $\left|D_{12}\right|$ and $\left|D_{21}\right|$ are both units so that the rank conditions (16) and the parity interlacing condition of Theorem 2.3 are automatically satisfied. We hence have the following result.

Corollary 2.2: Let $Z_{12}$ and $Z_{21}$ be stable transfer matrices and suppose that either $\operatorname{rank} Z_{12} \geq 2$ or $\operatorname{rank} Z_{21} \geq 2$. Then, $P 1$ is solvable.

Case 3: $Z$ is stabilizable and detectable from both Channels 1 and 2. This is the case where in (3), $P_{1} Q_{11}^{-1} R_{1}$ and $P_{2} Q_{11}^{-1} R_{2}$ are both bicoprime fractional representations of $Z_{11}$ and $Z_{22}$. The significance of this case is the application it finds in the multiple controller reliable synthesis problem of [10] (see Case 4 below).

Corollary 2.3: Let $P_{1} Q_{11}^{-1} R_{1}$ and $P_{2} Q_{11}^{-1} R_{2}$ be bicoprime fractions and suppose that (10) holds. Then, $\mathrm{P} 1_{i / o}$ is solvable if and only if $\left|Q_{11}\right|$ takes the same sign at the union of the real unstable blocking zeros of $Z_{12}$ and $Z_{21}$.

Proof: The proof is obtained by a specialization of the result of Theorem 2.2. We only outline the main steps. By the hypothesis that $P_{2} Q_{11}^{-1} R_{2}$ is bicoprime, we can let $C=I, D=I, \bar{D}=I$, and $\bar{R}_{1}=R_{1}$. It follows that

$$
\Lambda_{2} \Theta^{-1} \Gamma_{2}=R_{l} R_{1}\left[\Psi_{1}+\Psi_{2} P_{1}\left(E+G F_{r} R_{l}\right) R_{1}\right]^{-1} \Psi_{2} P_{1} P_{r} .
$$

Using the fact that $P_{1} Q_{11}^{-1} R_{1}$ is bicoprime and by various other coprimeness conditions, it is not difficult to show that the right-hand side of the above equality is also bicoprime. The next step is to establish the set equality

$$
\mathscr{S}=\left\{z \in \mathscr{R}_{+e} \mid P_{1} P_{r}=0 \text { or } R_{l} R_{1}=0\right\} .
$$

This can be shown by first noting that $P_{1} Q_{11}^{-1} R_{1}=D_{l}^{-1} N_{l}$ $=N_{r} D_{r}^{-1}$, in which each fraction is bicoprime, implies that $D_{l} P_{1}=\bar{P} Q_{11}, N_{l}=\bar{P} R_{1}, R_{1} D_{r}=Q_{11} \bar{R}$, and $N_{r}=$ $P_{1} \bar{R}$ for some matrices $\bar{P}, \bar{R}$ over $\mathscr{S}$. Now, using (4) and
(7), one can show that

$\operatorname{rank}\left[\begin{array}{ll}\Pi & \Gamma_{0}\end{array}\right](z)$

$$
=\left[\begin{array}{ccc}
I_{q} & \left(E+G F_{r} R_{l}\right) R_{1} & P_{r} \\
D_{l} P_{1} & N_{l} & 0
\end{array}\right](z)=q
$$

if and only if

$$
\operatorname{rank}\left[\begin{array}{ccc}
I_{q} & 0 & 0 \\
0 & P_{1} P_{r} H_{l} \bar{R} & P_{1} P_{r}
\end{array}\right](z)=q
$$

for any $z \in \mathscr{C}_{+e}$ which is the case if and only if $\left[P_{1} P_{r}\right](z)$ $=0$. Similarly, one can show that $\operatorname{rank}\left[\Pi^{\prime} \Lambda_{2}^{\prime}\right]^{\prime}(z)=q$ if and only if $\left[R_{l} R_{1}\right](z)=0$. This establishes the expression for $\mathscr{S}$ given above. It immediately follows that if $z \in \mathscr{S}$, then $\Lambda_{2} \Theta^{-1} \Gamma_{2}(z)=0$ by the bicoprime expression obtained above for the transfer matrix $\Lambda_{2} \Theta^{-1} \Gamma_{2}$. Hence, $\mathscr{S} \cap \mathscr{T}=\mathscr{S}$. Finally, by an easy computation, we have

$$
\begin{aligned}
|\Theta| & =\left|\Psi_{1}+\Psi_{2} P_{1}\left(G+P_{r} H_{l} E\right) R_{1}\right| \\
& =\left|\bar{\Psi}_{1}+P_{1}\left(E+G F_{r} R_{l}\right) R_{1} \bar{\Psi}_{2}\right|
\end{aligned}
$$

and

$$
\begin{gathered}
{\left[\Psi_{1}+\Psi_{2} P_{1}\left(G+P_{r} H_{l} E\right) R_{1}\right] D_{r}=I-\Psi_{2} P_{1} P_{r} H_{l} F P \bar{R}} \\
D_{l}\left[\bar{\Psi}_{1}+P_{1}\left(E+G F_{r} R_{l}\right) R_{1} \bar{\Psi}_{2}\right]=I-\bar{P} R H F_{r} R_{l} R_{1} \bar{\Psi}_{2}
\end{gathered}
$$

Noting that $\left|D_{l}\right|,\left|D_{r}\right|$, and $\left|Q_{11}\right|$ are associates, it follows that $|\Theta|$ takes the same sign at $\mathscr{S}$ if and only if so does $\left|Q_{11}\right|$. The result now follows by Theorem 2.2.

Case 4: Applications to full-feedback synthesis. The reliability of the closed-loop stability of a centralized (full) feedback system due to compensator breakdowns can be improved by using multiple stabilizing compensators in a decentralized structure [10]. The following problem is the multiple controller reliable synthesis problem (MCRSP) of Siljak [10] in our notation.

MCRSP: Let $Z=\left[Z_{i j}\right]$, where $Z_{i j} \in \boldsymbol{P}^{p_{i} \times r_{j}} i, j \in \mathbf{2}$, be the transfer matrix of a two-channel plant. Determine compensators $Z_{c i} \in \in^{r_{i} \times p} ; i \in 2$ such that: a) $\left(Z, \operatorname{diag}\left\{Z_{c 1}\right.\right.$, $\left.\left.Z_{c 2}\right\}\right)$ is internally stable; b) $\left(Z, \operatorname{diag}\left\{Z_{c 1}, 0\right\}\right)$ is internally stable; and c) $\left(Z, \operatorname{diag}\left\{0, Z_{c 2}\right\}\right)$ is internally stable.

The motivation of the problem is that (see also [10]) in the case of a possible breakdown of any one of the compensators, the other one continues to stabilize the plant $Z$. It is easily seen that the solution of the problem requires that $Z$ is stabilizable and detectable from both Channels 1 and 2 which implies the equivalence of the conditions b), c) above and i), ii) in the definition of $\mathrm{P} 1_{i / o}$. Consequently, MCRSP and $\mathrm{P} 1_{i / o}$ where $Z$ is stabilizable and detectable from Channels 1 and 2 are equivalent problems. We can then employ Corollary 2.3 to solve MCRSP.

Corollary 2.4: Suppose that either rank $Z_{12} \geq 2$ or rank $Z_{21} \geq 2$ and $Z_{11}, Z_{22}$ are strictly proper. Then, MCRSP is solvable if and only if $Z$ is stabilizable and detectable from both Channels 1 and 2 , and has an even 
number of real poles between each pair of zeros in the union of the sets of $\mathscr{R}_{+e}$-blocking zeros of $Z_{12}$ and $Z_{21}$.

It is possible to define and solve several different versions of the multiple controller reliable synthesis problem as applications of Corollary 2.3. As an example, we consider the following reliable full-feedback synthesis problem (RFFSP) in which the plant $Z$ has two input channels and only one output channel.

RFFSP: Let $G=\left[\begin{array}{ll}G_{1} & G_{2}\end{array}\right]$, where $G_{i} \in \boldsymbol{P}^{t \times s_{i}}, i \in \mathbf{2}$, be the transfer matrix of a two-input-channel, oneoutput-channel plant. Find a compensator $Z_{c}=\left[Z_{c 1}^{\prime}\right.$ $\left.Z_{c 2}^{\prime}\right]^{\prime}, Z_{c i} \in \boldsymbol{P}^{s_{i} \times i}, i \in \mathbf{2}$ such that: a) $\left(G, Z_{c}\right)$ is internally stable; b) $\left(G,\left[\begin{array}{ll}Z_{c 1}^{\prime} & 0\end{array}\right]^{\prime}\right)$ is internally stable; and c) $(G,[0$ $\left.\left.Z_{c 2}^{\prime}\right]\right)^{\prime}$ is internally stable.

Let $Q_{0}^{-1}\left[\begin{array}{ll}S_{1} & S_{2}\end{array}\right]$ be a left-coprime fractional representation of $G$. For RFFSP to be solvable, it is clear that $G$ must be stabilizable from both of the input channels separately, in other words $\left(Q_{0}, S_{1}\right)$ and $\left(Q_{0}, S_{2}\right)$ must be left coprime pairs. Now consider the two-input, twooutput expanded plant

$$
G_{e}=\left[\begin{array}{ll}
G_{1} & G_{2} \\
G_{1} & G_{2}
\end{array}\right]=\left[\begin{array}{l}
I \\
I
\end{array}\right] Q_{0}^{-1}\left[\begin{array}{ll}
S_{1} & S_{2}
\end{array}\right] .
$$

Assume that either rank $G_{1} \geq 2$ or rank $G_{2} \geq 2$. Applying Corollary 2.3 to $Z=G_{e}$ with $Z_{11}=G_{1}, Z_{12}=G_{2}, Z_{21}=$ $G_{1}$, and $Z_{22}=G_{2}$, one can obtain $Z_{c 1}, Z_{c 2}$ which solve P1 for the expanded plant $G_{e}$, if $\left|Q_{0}\right|$ takes the same sign at $\left\{z \in \mathscr{R}_{+e} \mid G_{1}=0\right.$ or $\left.G_{2}=0\right\}$. By the fact that $\left(Q_{0}, S_{1}\right)$ and $\left(Q_{0}, S_{2}\right)$ are coprime pairs in (18), it holds that: i) $\left(G_{e}\right.$, $\left.\operatorname{diag}\left\{Z_{c 1}, 0\right\}\right)$ is internally stable; ii) $\left(G_{e}, \operatorname{diag}\left\{0, Z_{c 2}\right\}\right)$ is internally stable; and iii) $\left(G_{e}, \operatorname{diag}\left\{Z_{c 1}, Z_{c 2}\right\}\right)$ is internally stable. It can be verified that i), ii), and iii) imply a), b), and $\mathrm{c}$ ). As a result, we have the following.

Suppose that either rank $G_{1} \geq 2$ or rank $G_{2} \geq 2$, and $G$ is strictly proper. Then, a sufficient condition for RFFSP to be solvable is that $G$ has an even number of real poles between each pair of zeros in the union of the sets of $\mathscr{R}_{+e}$-blocking zeros of $G_{1}$ and $G_{2}$.

\section{FeEdback Interconnected Systems}

Given the feedback interconnected system $\bar{\Sigma}$ of Fig. 2, where $T_{11} \in \boldsymbol{P}^{p_{1} \times r_{1}}, T_{22} \in \boldsymbol{P}^{p_{2} \times r_{2}}, T_{12} \in \boldsymbol{P}^{r_{1} \times p_{2}}, T_{21} \in$ $\boldsymbol{P}^{r_{2} \times p_{1}}$ with $T_{11}, T_{22}$ strictly proper, let

$$
T_{i j}=D_{i j}^{-1} N_{i j}=\bar{N}_{i j} \bar{D}_{i j}^{-1}
$$

be some left- and right-coprime fractional representations of the subplant transfer matrices for $i, j \in \mathbf{2}$. Then the transfer matrix $T$ of $\bar{\Sigma}$ has the fractional representation

$$
\begin{aligned}
& T=\left[\begin{array}{llll}
I & 0 & 0 & 0 \\
0 & 0 & 0 & I
\end{array}\right]\left[\begin{array}{cccc}
D_{11} & -N_{11} & 0 & 0 \\
0 & D_{12} & 0 & -N_{12} \\
-N_{21} & 0 & D_{21} & 0 \\
0 & 0 & -N_{22} & D_{22}
\end{array}\right]^{-1} \\
& \cdot\left[\begin{array}{cc}
N_{11} & 0 \\
0 & 0 \\
0 & 0 \\
0 & N_{22}
\end{array}\right]
\end{aligned}
$$

Let

$$
\begin{gathered}
Q_{11}:=\left[\begin{array}{cccc}
D_{11} & -N_{11} & 0 & 0 \\
0 & D_{12} & 0 & -N_{12} \\
-N_{21} & 0 & D_{21} & 0 \\
0 & 0 & -N_{22} & D_{22}
\end{array}\right] \\
R_{1}:=\left[\begin{array}{c}
N_{11} \\
0 \\
0 \\
0
\end{array}\right], R_{2}:=\left[\begin{array}{c}
0 \\
0 \\
0 \\
N_{22}
\end{array}\right] \\
P_{1}:=\left[\begin{array}{llll}
I & 0 & 0 & 0
\end{array}\right], P_{2}:=\left[\begin{array}{llll}
0 & 0 & 0 & I
\end{array}\right] .
\end{gathered}
$$

The system $\bar{\Sigma}$ is free of unstable hidden modes if and only if the fraction (19) is bicoprime, i.e., $\left(Q_{11}, R_{1}, R_{2}\right)$ is left coprime and $\left(P_{1}, P_{2}, Q_{11}\right)$ is right coprime, which in turn is the case if and only if

$$
\left(N_{22}, D_{21}\right),\left(N_{11}, D_{12}\right) \text { are right-coprime pairs }
$$

and

$$
\left(\bar{D}_{21}, \bar{N}_{11}\right),\left(\bar{D}_{12}, \bar{N}_{22}\right) \text { are left-coprime pairs. }
$$

Let

$$
\bar{D}_{21}^{-1} \bar{N}_{11}=\hat{N}_{11} \hat{D}_{21}^{-1}, \bar{D}_{12}^{-1} \bar{N}_{22}=\hat{N}_{22} \hat{D}_{12}^{-1}
$$

for some right-coprime pairs $\left(\hat{N}_{11}, \hat{D}_{21}\right)$ and $\left(\hat{N}_{22}, \hat{D}_{12}\right)$. The system $\bar{\Sigma}$ is free of unstable decentralized fixed modes if and only if the coprimeness conditions (21), (22) hold and for all $z \in \mathscr{C}_{+e}$ it holds that

$$
\begin{array}{r}
\operatorname{rank}\left[\begin{array}{ccc}
D_{12} & N_{12} \bar{N}_{22} & 0 \\
-N_{11} & 0 & 0 \\
0 & 0 & D_{21}
\end{array}\right] \geq r_{1}+r_{2} \\
\operatorname{rank}\left[\begin{array}{ccc}
D_{21} & N_{21} \bar{N}_{11} & 0 \\
-N_{22} & 0 & 0 \\
0 & 0 & D_{12}
\end{array}\right] \geq r_{1}+r_{2} .
\end{array}
$$

Exploiting the fact that $\left(\bar{N}_{22}, \bar{D}_{12}\right)$ and $\left(\bar{N}_{11}, \bar{D}_{21}\right)$ are left-coprime pairs by (22), it can be seen that these rank inequalities hold if and only if for all $z \in \mathscr{C}_{+e}$ the simpler inequalities

$$
\begin{aligned}
& \operatorname{rank}\left[\begin{array}{cc}
D_{21} & 0 \\
0 & N_{11} \bar{N}_{12} \hat{N}_{22}
\end{array}\right] \geq r_{2} \\
& \operatorname{rank}\left[\begin{array}{cc}
D_{12} & 0 \\
0 & N_{22} \bar{N}_{21} \hat{N}_{11}
\end{array}\right] \geq r_{1}
\end{aligned}
$$

hold. The main result of this section can now be given. Consider the condition

$$
\operatorname{rank}\left(T_{11} T_{12} T_{22}\right) \geq 2 \text { or } \operatorname{rank}\left(T_{22} T_{21} T_{11}\right) \geq 2 \text {. }
$$

Theorem 3.1: Suppose that (24) holds. Then P2 has a solution if and only if $\bar{\Sigma}$ has no unstable decentralized fixed modes (equivalently, the coprimeness conditions (21), (22) and the rank conditions (23) hold) and $\left|D_{12}\right|\left|D_{21}\right|$ 
takes the same sign at the set

$\overline{\mathscr{S}}=\left\{\mathscr{R}_{+e}\right.$-blocking zeros of $\left.N_{11} T_{12} \bar{N}_{22}\right\}$

$\cup\left\{\mathscr{R}_{+}\right.$-blocking zeros of $\left.N_{22} T_{21} \bar{N}_{11}\right\}$.

Proof: Observe that $T_{i i} T_{i j} T_{i j}=D_{i i}^{-1} N_{i i} \bar{N}_{i j} \hat{N}_{i j}$ $\left(\bar{D}_{j j} \hat{D}_{i j}\right)^{-1}$ for $i, j \in \mathbf{2}, i \neq j$ and hence by (24) we have

$$
\operatorname{rank}\left(N_{11} \bar{N}_{12} \hat{N}_{22}\right) \geq 2 \text { or rank }\left(N_{22} \bar{N}_{21} \hat{N}_{11}\right) \geq 2 \text {. }
$$

Also let $K_{i}, L_{i}, \bar{K}_{i}, \bar{L}_{i}, i \in \mathbf{2}$ be such that

$$
\begin{aligned}
& {\left[\begin{array}{cc}
D_{11} & N_{11} \\
-\bar{L}_{1} & \bar{K}_{1}
\end{array}\right]\left[\begin{array}{cc}
\bar{D}_{21} K_{1} & -\bar{N}_{11} \\
L_{1} & \bar{D}_{11}
\end{array}\right]=I,} \\
& {\left[\begin{array}{cc}
D_{22} & N_{22} \\
-\bar{L}_{2} & \bar{K}_{2}
\end{array}\right]\left[\begin{array}{cc}
\bar{D}_{12} K_{2} & -\bar{N}_{22} \\
L_{2} & \bar{D}_{22}
\end{array}\right]=I .}
\end{aligned}
$$

Such matrices exist by left coprimeness of the pairs $\left(D_{11} \bar{D}_{21}, N_{11}\right)$ and $\left(D_{22} \bar{D}_{12}, N_{22}\right)$. The set of all internally stabilizing compensators $Z_{c 1}\left(Z_{c 2}\right)$ of $T_{11}\left(T_{22}\right)$ can then be written as

$$
\begin{aligned}
Z_{c 1}=\left(L_{1}+\bar{D}_{11} X_{1}\right)\left(\bar{D}_{21} K_{1}-\bar{N}_{11} X_{1}\right)^{-1} \\
Z_{c 2}=\left(L_{2}+\bar{D}_{22} X_{2}\right)\left(\bar{D}_{12} K_{2}-\bar{N}_{22} X_{2}\right)^{-1}
\end{aligned}
$$

for some $X_{i} \in S^{r_{i} \times p_{i}}, i \in 2$. Moreover, for any such $Z_{c i}$, the pair of plants $\left(T, \operatorname{diag}\left\{Z_{c 1}, Z_{c 2}\right\}\right)$ is internally stable if and only if

$$
\Theta:=\left[\begin{array}{c}
D_{12} \\
-N_{21}\left(\bar{D}_{21} K_{1}-\bar{N}_{11} X_{1}\right) N_{11}
\end{array}\right.
$$

is a unimodular matrix for some $X_{1}$ and $X_{2}$. This follows on performing simple unimodular operations on the closed-loop denominator matrix

$$
\left[\begin{array}{ccc}
Q_{11} & R_{1} P_{c 1} & R_{2} P_{c 2} \\
-P_{1} & Q_{c 1} & 0 \\
-P_{2} & 0 & Q_{c 2}
\end{array}\right]
$$

where the various matrices are as given in (20) and (27). Summarizing our considerations so far if $\bar{\Sigma}$ is free of unstable decentralized fixed modes, then $\mathrm{P} 2$ has a solution if and only if there exist matrices $X_{1}$ and $X_{2}$ such that the matrix $\Theta$ is unimodular. We now find an equivalent matrix $\tilde{\Theta}$ to $\Theta$ which is in a more convenient form. For this purpose, let $\left(\tilde{D}_{12}, \tilde{N}_{11}\right)$ and $\left(\tilde{D}_{21}, \tilde{N}_{22}\right)$ be some right-coprime pairs such that $\left|D_{12}\right|=\left|\tilde{D}_{12}\right|,\left|D_{21}\right|=\left|\tilde{D}_{22}\right|$ and

$$
N_{11} D_{2}^{-1}=\tilde{D}_{12}^{-1} \tilde{N}_{11}, N_{22} D_{21}^{-1}=\tilde{D}_{21}^{-1} \tilde{N}_{22} .
$$

Consider

$$
\tilde{\Theta}:=\left[\begin{array}{cc}
\tilde{D}_{12} & -\tilde{N}_{11} N_{12}\left(\bar{D}_{12} K_{2}-\bar{N}_{22} X_{2}\right) \\
-\tilde{N}_{22} N_{21}\left(\bar{D}_{21} K_{1}-\bar{N}_{11} X_{1}\right) & \tilde{D}_{21}
\end{array}\right] .
$$

It easily follows that

$$
\operatorname{diag}\left\{\tilde{N}_{11}, \tilde{N}_{22}\right\} \Theta=\tilde{\Theta} \operatorname{diag}\left\{N_{11}, N_{22}\right\}
$$

From the various coprimeness conditions above, it also follows that $\tilde{\Theta}$ is unimodular if and only if $\Theta$ is unimodular.

[Only If] If P2 has a solution, then $\bar{\Sigma}$ is free of decentralized fixed modes so that the coprimeness conditions (21), (22) as well as the rank conditions (23) hold. Moreover, the compensator transfer matrices are necessarily of the form (27) for some $X_{1}$ and $X_{2}$, since the compensators separately satisfy the conditions i) and ii) of the problem definition. By the condition iii), the compensators also achieve decentralized stabilization so that $X_{1}$ and $X_{2}$ are such that $|\tilde{\Theta}|$ is a unit, or equivalently, it has constant sign at all points of $\mathscr{R}+e$. By unimodularity of $\tilde{\Theta}$, the pair $\left(\tilde{D}_{12}, \bar{N}_{11} N_{12}\left(\bar{D}_{12} K_{2}-\bar{N}_{22} X_{2}\right)\right)$ is left coprime which implies that the pair $\left(\hat{D}_{12}, X_{2}\right)$ is left coprime. Let

$$
\hat{D}_{12}^{-1} X_{2}=B_{2} A_{12}^{-1}
$$

for some right-coprime pair $\left(B_{2}, A_{12}\right)$ such that $\left|A_{12}\right|=$ $\left|\tilde{D}_{12}\right|$. The following alternative expression for $|\tilde{\Theta}|$ is now easy to verify also using (26)

$$
\begin{aligned}
|\tilde{\Theta}|=\mid \tilde{D}_{21} A_{12}-\tilde{N}_{22} N_{21} \bar{N}_{11}\left(\bar{K}_{1}-\right. & \left.X_{1} N_{11}\right) \\
& \cdot \bar{N}_{12}\left(K_{2} A_{12}-\hat{N}_{22} B_{2}\right) \mid .
\end{aligned}
$$

When this is evaluated at any $\mathscr{R}_{+e}$-blocking zero of $\tilde{N}_{22} N_{21} \bar{N}_{11}$, one has $|\tilde{\Theta}|=\left|\tilde{D}_{21}\right|\left|A_{12}\right|=\left|\tilde{D}_{21}\right| \tilde{D}_{12} \mid$. This

$$
\left.\begin{array}{c}
-N_{12}\left(\bar{D}_{12} K_{2}-\bar{N}_{22} X_{2}\right) N_{22} \\
D_{21}
\end{array}\right]
$$

yields that $\left|D_{21}\right|\left|D_{12}\right|$ has constant sign at all $\mathscr{R}_{+}$-blocking zeros of $N_{22} N_{21} \bar{N}_{11}$. By a completely dual argument, one can establish that at any $\mathscr{R}_{+e}$-blocking zero of $\bar{N}_{11} N_{12} \bar{N}_{22}$ one has $|\tilde{\Theta}|=\left|\tilde{D}_{12}\right|\left|\tilde{D}_{21}\right|$. Consequently, at all zeros in the set

$\overline{\mathscr{S}}:=\left\{\mathscr{R}_{+e}\right.$-blocking zeros of $\left.\bar{N}_{11} N_{12} \bar{N}_{22}\right\}$

$$
\cup\left\{\mathscr{R}_{+e} \text {-blocking zeros of } \tilde{N}_{22} N_{21} \bar{N}_{11}\right\}
$$

the sign of $\left|\tilde{D}_{12}\right|\left|\tilde{D}_{21}\right|$ is constant. Since the latter is an associate of $\left|D_{12}\right|\left|D_{21}\right|$, the sign of $\left|D_{12}\right|\left|D_{21}\right|$ remains constant for all zeros in $\overline{\mathscr{S}}$. Finally note that

$$
\begin{aligned}
& N_{11} \bar{N}_{12} \hat{N}_{22} \hat{D}_{12}^{-1}=\tilde{D}_{12}^{-1} \tilde{N}_{11} N_{12} \bar{N}_{22} \\
& N_{22} \bar{N}_{21} \hat{N}_{11} \hat{D}_{21}^{-1}=\tilde{D}_{21}^{-1} \tilde{N}_{22} N_{21} \bar{N}_{11}
\end{aligned}
$$

where each fraction is coprime. Consequently, the $\mathscr{R}_{+e^{-}}$ blocking zeros of $N_{11} \bar{N}_{12} \hat{N}_{22}$ (respectively, $N_{22} \bar{N}_{21} \hat{N}_{11}^{+e}$ ) 
are the same as the $\mathscr{R}_{+e}$-blocking zeros of $\tilde{N}_{11} N_{12} \bar{N}_{22}$ (respectively, $\tilde{N}_{22} N_{21} \bar{N}_{11}$ ). In other words, the set $\overline{\mathscr{S}}$ has the alternative descriptions

$\overline{\mathscr{S}}:=\left\{\mathscr{R}_{+e}\right.$-blocking zeros of $\left.N_{11} \bar{N}_{12} \hat{N}_{22}\right\}$

$\cup\left\{\mathscr{R}_{+e}\right.$-blocking zeros of $\left.N_{22} \bar{N}_{21} \hat{N}_{11}\right\}$

or the one in the theorem statement. The necessity of the parity interlacing condition in the theorem statement is thus established.

[If] Let the coprimeness conditions (21), (22), and the rank conditions (16) hold. We need to show that the parity interlacing condition of the theorem, under the hypothesis (25) implies the existence of $X_{1}, X_{2}$ such that $\tilde{\Theta}$ is unimodular. We first show that there exists $X_{1}$ satisfying the following three conditions, where $Y_{1}:=\bar{D}_{21} K_{1}-$ $\bar{N}_{11} X_{1}$.

C1: $\left(\tilde{D}_{21}, \tilde{N}_{22} N_{21} Y_{1}\right)$ is left coprime.

C2: $\left(\tilde{N}_{22} N_{21} Y_{1}, \tilde{D}_{12}\right)$ is left coprime.

C3: The set of $\mathscr{R}_{+e}$-blocking zeros of $\tilde{N}_{22} N_{21} Y_{1}$ $N_{11} \bar{N}_{12} \hat{N}_{22}$ is the set $\overline{\mathscr{S}}_{\text {. }}$

To show that such an $X_{1}$ exists, we show that the set of $X_{1}$ satisfying each condition is open and dense in $S^{r_{1} \times p_{1}}$. Note, by using $\tilde{D}_{21}^{-1} \tilde{N}_{22} N_{21}=N_{22} \bar{N}_{21} \bar{D}_{21}^{-1}$ and $\bar{D}_{21}^{-1} \bar{N}_{11}=$ $\hat{N}_{11} \hat{D}_{21}^{-1}$ where each fraction is coprime, that $\mathrm{C} 1$ holds iff $\left(\hat{D}_{21}, X_{1}\right)$ is left coprime. This last condition holds for almost all $X_{1} \in S^{r_{1} \times p_{1}}$. On the other hand, the first rank inequality of (23) holds for all $z \in \mathscr{C}_{+e}$ iff for all such $z$ one has

$$
\operatorname{rank}\left[\begin{array}{cc}
\tilde{D}_{12} & \tilde{N}_{22} N_{21} \bar{D}_{21} K_{1} \\
0 & \tilde{N}_{22} N_{21} \bar{N}_{11}
\end{array}\right] \geq p_{1}
$$

as the two relevant matrices can be shown to be equivalent over $S$. This last rank condition is equivalent to the quadruple $\left(0, \tilde{D}_{12}, \tilde{N}_{22} N_{21} \bar{D}_{21} K_{1}, \tilde{N}_{22} N_{21} \bar{N}_{11}\right)$ being complete. Applying [7, lemma 2.6], it now follows that $\mathrm{C} 2$ also holds for almost all $X_{1} \in \boldsymbol{S}^{r_{1} \times p_{1}}$. Finally, by Lemma A.2 of the Appendix and by the hypothesis (25), it follows that the condition $\mathrm{C} 3$ is satisfied for almost all $X_{1} \in S^{r_{1} \times p_{1}}$. Therefore, there exists an open and dense subset of $\boldsymbol{S}^{r_{1} \times p_{1}}$ every element $X_{1}$ of which satisfies all three conditions C1-C3. Fix one such $X_{1}$ and $Y_{1}$ and let $A, B, \bar{A}, \bar{B}$ be some matrices satisfying $|A|=\left|\tilde{D}_{12}\right|$ and

$$
\left[\begin{array}{cc}
\bar{A} & -\bar{B} \\
B & A
\end{array}\right]\left[\begin{array}{c}
\tilde{D}_{12} \\
-\tilde{N}_{22} N_{21} Y_{1}
\end{array}\right]=\left[\begin{array}{l}
I \\
0
\end{array}\right]
$$

which is possible by $\mathrm{C} 2$. On multiplying $\tilde{\Theta}$ on the left by the first matrix above, it is clear that

$$
|\tilde{\Theta}|=\left|A \tilde{D}_{21}-B \tilde{N}_{11} N_{12}\left(\bar{D}_{12} K_{2}-\bar{N}_{22} X_{2}\right)\right|
$$

or noting that $B \bar{N}_{11} N_{12} \bar{D}_{12}=A \bar{N}_{22} N_{21} Y_{1} N_{11} \bar{N}_{12}$ and $Y_{1} N_{11}=\bar{N}_{11} \bar{Y}_{1}$ with $\bar{Y}_{1}:=\bar{K}_{1}-X_{1} N_{11}$

$$
\begin{aligned}
|\tilde{\Theta}| & =\left|\hat{A}+\hat{B} X_{2}\right| \\
& =\left|A\left(\tilde{D}_{21}-\tilde{N}_{22} N_{21} \bar{N}_{11} \bar{Y}_{1} \bar{N}_{12} K_{2}\right)+B \tilde{N}_{11} N_{12} \bar{N}_{22} X_{2}\right|
\end{aligned}
$$

where the matrices $\hat{A}, \hat{B}$ have the obvious definitions. By $\mathrm{C} 1$ and various other left coprimeness conditions, it follows that $(\hat{A}, \hat{B})$ is left coprime. From (26) it follows that $\bar{K}_{2}=\hat{D}_{12} \hat{K}_{2}$, for some $\hat{K}_{2}$. On noting that $\left(\tilde{D}_{21}-\right.$ $\left.\tilde{N}_{22} N_{21} \bar{N}_{11} \quad \bar{Y}_{1} \bar{N}_{12} K_{2}\right)^{-1} \tilde{N}_{22}=N_{22}\left(D_{21}-N_{21} Y_{1} N_{11}\right.$ $\left.\bar{N}_{12} \hat{N}_{22} \hat{K}_{2}\right)^{-1}$ where both fractions are coprime, and $\left|D_{21}\right|=\left|\tilde{D}_{21}\right|$, it follows that $|\hat{A}|$ takes the same sign at all $z \in \overline{\mathscr{S}}$. Also from $\mathrm{C} 3$, the set of $\mathscr{R}+e^{\text {-blocking zeros of } \hat{B}}$ is precisely the set $\overline{\mathscr{S}}$. The parity interlacing condition of the theorem statement now implies that $|\hat{A}|$ has constant

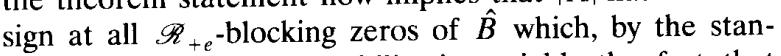
dard result on strong stabilization, yields the fact that there exists $X_{2}$ such that $\tilde{\Theta}$ is unimodular.

Remark 3.1: The connectivity assumption of the theorem is only used in the 'If' part of the proof. Hence, all systems $\bar{\Sigma}$ for which P2 has a solution satisfy the given parity interlacing condition.

Remark 3.2: On comparing the solvability conditions to problems $\mathrm{P} 1$ and $\mathrm{P} 2$, it is clear that the poles and zeros of the main subplants play a dual role. This is certainly not a coincidence and is a consequence of a deeper duality in the feedforward and feedback interconnections of Figs. 1 and 2 , or in the problem definitions P1 and P2. We have at present no precise formulation of this latter duality but can offer the following crude version. Assuming the main subplant transfer matrices $Z_{i i}, T_{i i}$ for $i \in \mathbf{2}$ are all nonsingular, it is easy to verify, via (1) and (2), that

$$
T^{-1}=Z\left[T_{11}^{-1},-T_{12}, T_{22}^{-1},-T_{21}\right]=\left[\begin{array}{cc}
T_{11}^{-1} & -T_{12} \\
-T_{21} & T_{22}^{-1}
\end{array}\right]
$$

where the notation $Z\left[Z_{11}, Z_{12}, Z_{22}, Z_{21}\right]$ is a more explicit representation for $Z$ as a function of its subplant transfer matrices. (Similarly, $Z^{-1}=T\left[Z_{11}^{-1},-Z_{12}, Z_{22}^{-1}\right.$, $\left.-Z_{21}\right]$.) Neglecting all the properness requirements and assuming that both compensators employed are nonsingular, it follows that $\left(Z_{c 1}, Z_{c 2}\right)$ is a solution to $\mathrm{P} 2$ for $T$ if and only if $\left(Z_{c 1}^{-1}, Z_{c 2}^{-1}\right)$ is a solution to $\mathrm{P} 1$ for $T^{-1}$. Consequently, $\mathrm{P} 2$ is solvable for $T$ if and only if $\mathrm{P} 1$ is solvable for $T^{-1}$, and similarly, $\mathrm{P} 1$ is solvable for $Z$ if and only if $\mathrm{P} 2$ is solvable for $Z^{-1}$. A refinement of the formulation of this type of duality between P1 and P2 will be useful in unifying the reliable synthesis problems for $N$-channel $(N \geq 2)$ interconnected plants.

We now specialize the result of Theorem 3.1 to two cases.

Case 1: $T_{11}$ and $T_{22}$ are stable transfer matrices. In this case, we can let $D_{i i}=I, \bar{D}_{i i}=I$ for $i \in \mathbf{2}$ so that $N_{11} \bar{N}_{12} \hat{N}_{22}$ and $T_{11} T_{12} T_{22}$ have the same set of $\mathscr{R}_{+e^{-}}$ blocking zeros. Similarly for their duals.

Corollary 3.1: Let $T_{11}$ and $T_{22}$ be stable transfer matrices and suppose that (24) holds. Then, P2 has a solution if and only if the conditions (21), (22), and (23) hold and $\left|D_{12}\right|\left|D_{21}\right|$ takes the same sign at the union of the $\mathscr{R}_{+} e^{-}$ blocking zeros of $T_{11} T_{12} T_{22}$ and $T_{22} T_{21} T_{11}$.

Case 2: $T_{12}$ and $T_{21}$ are stable transfer matrices. In this 
case $\left|D_{12}\right|,\left|D_{21}\right|$ are units and the parity interlacing condition as well as the coprimeness and rank conditions (21) (22), and (23) are all satisfied.

Corollary 3.2: Let $T_{12}$ and $T_{21}$ be stable transfer matrices and suppose that $(24)$ holds. Then, P2 is solvable.

\section{CONCLUSIONS}

We have obtained conditions for the solvability of problems $\mathrm{P} 1, \mathrm{P} 1_{i / o}, \mathrm{P} 2$, and a number of other reliable decentralized stabilization problems under mild connectivity assumptions (10) and (24). The connectivity conditions arise as a consequence of our particular technique of compensator construction and are not believed to be inherently related to solvability. The fact that the central requirement for reliable decentralized stabilization in all these problems is a parity interlacing property indicates that reliable synthesis is generically easy to achieve. The main results obtained in the paper also indicate some interesting features of feedforward and feedback interconnected plants that admit reliable decentralized synthesis. As far as reliability is concerned:

1) stable interconnections cause no extra problems;

2) stable main subplants lead to considerable simplification in $\mathrm{P} 1$;

3) nonsingular and minimum-phase main subplants lead to considerable simplifications in $\mathrm{P} 2$;

4) periodically time-varying feedbacks should help since these lead to an elimination of blocking zeros (see [4]);

5) the roles played by the poles and zeros of the main subplants are dual in feedforward and feedback interconnected systems.

We note that one can think of many quite different extensions of the problems we cosidered to the case of $N$-channels for $N>2$. In all these extensions, the general features noted in 1)-5) are anticipated to find a fundamental use.

\section{APPENDIX}

In this Appendix we prove Proposition 2.3. We need the following technical lemmata.

Lemma A.1: Let $\tilde{D} \in \boldsymbol{S}^{\tilde{p} \times \bar{r}}, \tilde{E} \in \boldsymbol{S}^{\bar{p} \times \tilde{n}}, \tilde{F} \in \boldsymbol{S}^{\tilde{m} \times \bar{r}}$, and $X_{0} \in S^{\tilde{n} \times \bar{m}}$ where $\tilde{p} \geq 2, \tilde{r} \geq 2$. Let $q_{0}$ be an integer satisfying $0<q_{0}<\min (\tilde{p}, \tilde{r})$ such that $\operatorname{rank}\left(\tilde{D}+\tilde{E} X_{0} \tilde{F}\right)$ $\geq q$, for all $z \in \mathscr{C}_{ \pm e}$. Then, given $z_{0} \in \mathscr{C}_{+e}$, the class of $\Delta$ for which $\operatorname{rank}\left(D+\tilde{E}\left(X_{0}+\Delta\right) \tilde{F}\right)\left(z_{0}\right)>q_{0}$ is dense in $S^{\tilde{n} \times \tilde{m}}$ if and only if

$$
\operatorname{rank}\left[\begin{array}{cc}
\tilde{D} & \tilde{E}
\end{array}\right]\left(z_{0}\right)>q_{0} \text { and } \operatorname{rank}\left[\begin{array}{ll}
\tilde{D}^{\prime} & \tilde{F}^{\prime}
\end{array}\right]^{\prime}\left(z_{0}\right)>q_{0} .
$$

Lemma A.2: Let $\tilde{A} \in \boldsymbol{S}^{\tilde{p} \times \bar{r}}, B \in \boldsymbol{S}^{\bar{p} \times \bar{n}}$, and $C \in \boldsymbol{S}^{\tilde{m} \times \bar{r}}$ be such that either rank $\tilde{B} \geq 2$ or rank $\tilde{C} \geq 2$. Then, for $z \in \mathscr{C}_{+e},(\tilde{A}+\tilde{B} X \tilde{C})=0$ only if $[\tilde{A} \quad \tilde{B}]=0$ or $\left[\tilde{A}^{\prime}\right.$ $\left.\tilde{C^{\prime}}\right]^{\prime}=0$, for almost all $X \in \mathscr{X}$.

Proof of Proposition 2.3: Proof of i) is immediate, and is therefore omitted. To prove ii), define $\boldsymbol{Q}_{11}=\{z \in$ $\mathscr{C}_{+e}|| Q_{11} \mid=0$ ) and $\tilde{Q}_{11}=Q_{11} \cap \mathscr{S}$. We will first show that if $X_{0} \in \mathscr{X}$ is such that $\mathscr{S} \nsubseteq \mathscr{S}\left(X_{0}\right)$, there exists a perturbation on $X_{0}$, which provides $\mathscr{S}=\mathscr{S}\left(X_{p}\right)$, where $X_{p}=$ $X_{0}+\Delta$ for some $\Delta$. Moreover, the norm of $\Delta$ can be chosen smaller than any prespecified positive number. Let $z_{0} \in \boldsymbol{Q}_{11}-\tilde{\boldsymbol{Q}}_{11}$. Then, $\operatorname{rank}\left[\Pi \Gamma_{0}\right]>q$ and $\operatorname{rank}\left[\Pi^{\prime}\right.$ $\left.\Lambda_{2}^{\prime}\right]^{\prime}>q$. Applying Lemma A.1, with $\tilde{D}=\Pi, \tilde{E}=\Gamma_{0}$, and $\tilde{F}=\Lambda_{2}$, we have that the class of $X$ satisfying $z_{0} \notin \mathscr{S}(X)$ is dense in $\mathscr{X}$. (Recall that $\mathscr{X}$ is dense in $\mathscr{S}^{r_{2} \times p_{2}}$.) It can also be verified that this class is open. Hence, if $\mathscr{S}\left(X_{0}\right)$ contains any $z$ from $Q_{11}-\tilde{Q}_{11}$, there exists $\Delta_{0}$ with arbitrarily small norm satisfying $\mathscr{S}\left(X_{0}+\right.$ $\left.\Delta_{0}\right) \cap\left(Q_{11}-\tilde{Q}_{11}\right)=\emptyset$, i.e., $\mathscr{S}\left(X_{0}+\Delta_{0}\right)$ does not contain any zero from $Q_{11}-\tilde{Q}_{11}$.

Define $A(X)=E+\left(G F_{r}-P_{r} X\right) R_{l}$. Let $D_{l} P_{1} C^{-1}=$ $T_{l}^{-1} M_{l}$, for a left-coprime pair of matrices $\left(T_{l}, M_{l}\right)$. Note that rank $Z_{21}=\operatorname{rank} R_{l} \bar{R}_{1}$ and rank $Z_{12}=\operatorname{rank} M_{l} P_{r}$ and we have that either rank $R_{l} \bar{R}_{1} \geq 2$ or rank $M_{l} P_{r} \geq 2$. For any $X \in \mathscr{X}, \mathscr{R}(X)=\left\{z \in \mathscr{R}_{+e}-Q_{11} \mid\left(-T_{l} N_{l} \bar{D}+\right.\right.$ $\left.\left.M_{l} A(X) \bar{R}_{1}\right)=0\right\}=\mathscr{S}(X)-Q_{11}$. Observe that, if $z \in$ $\mathscr{R}_{+e}-\boldsymbol{Q}_{11}$ satisfies

$$
\left[-T_{l} N_{l} \bar{D}+M_{l}\left(E+G F_{r} R_{l}\right) \bar{R}_{1}-M_{l} P_{r}\right]=0
$$

or

$$
\left[\begin{array}{c}
-T_{l} N_{l} \bar{D}+M_{l}\left(E+G F_{r} R_{l}\right) \bar{R}_{1} \\
R_{l} \bar{R}_{1}
\end{array}\right]=0
$$

then, $z \in \mathscr{K}(X)$, independent of $X$. Conversely, Lemma A.2 implies that for almost $X \in \mathscr{X}, \mathscr{K}(X)$ consists only of those $z$, for which (28) and (29) hold. On the other hand, for $z \in \mathscr{R}_{+e}-Q_{11}$, (28) holds only if $\operatorname{rank}\left[\begin{array}{ll}\Pi & \Gamma_{0}\end{array}\right]=q$ and (29) holds only if $\operatorname{rank}\left[\begin{array}{ll}\Pi^{\prime} & \Lambda_{2}^{\prime}\end{array}\right]^{\prime}=q$. That is, there exists $\Delta_{1}$ with arbitrarily small norm such that $z \in \mathscr{P}\left(X_{0}\right.$ $\left.+\Delta_{0}+\Delta_{1}\right)-Q_{11}$ implies $\operatorname{rank}\left[\Pi \Gamma_{0}\right]=q$ or $\operatorname{rank}\left[\Pi^{\prime}\right.$ $\left.\Lambda_{2}^{\prime}\right]^{\prime}=q$ holds. The norm of $\Delta_{1}$ can be chosen sufficiently small still to have $\mathscr{S}\left(X_{0}+\Delta_{0}+\Delta_{1}\right) \cap\left(Q_{11}-\tilde{Q}_{11}\right)=\emptyset$. These results imply $\mathscr{S}\left(X_{0}+\Delta\right)=\mathscr{S}$, where $\Delta=\Delta_{0}+\Delta_{1}$. Also, the norm of $\Delta$ can be chosen smaller than any prespecified positive number, by restricting the norms of $\Delta_{0}$ and $\Delta_{\mathrm{l}}$ suitably.

It will now be shown that if $X_{0} \in \mathscr{X}$ satisfies $\mathscr{S}\left(X_{0}\right)=$ $\mathscr{S}$, there exists an open ball around $X_{0}$ such that for all $X$ belonging to this ball $\mathscr{S}(X)=\mathscr{S}$. First, one can verify that the class of $X$ satisfying $\mathscr{P}(X) \cap\left(Q_{11}-\tilde{Q}_{11}\right)=\emptyset$ is open in $\mathscr{X}$. Clearly, $\mathscr{S}\left(X_{0}\right) \cap\left(Q_{11}-\tilde{Q}_{11}\right)=\emptyset$, and there exists an open ball around $X_{0}$ such that for all $X \in \mathscr{X}$ in this ball, $\mathscr{P}(X) \cap\left(Q_{11}-\tilde{Q}_{11}\right)=\emptyset$. Also, $\mathscr{S}\left(X_{0}\right)-Q_{11}$ consists only of those $z$ for which (28) or (29) holds. From Lemma A.2, there exists an open ball around $X_{0}$ such that for all $X \in \mathscr{Z}$ in this ball, $\mathscr{A}\left(X_{0}\right)-Q_{11}$ consists only of those $z$ for which (28) or (29) holds. Since the intersection of open sets is open, we conclude that there is an open ball around $X_{0}$, such that for all $X$ in this ball $\mathscr{S}(X) \cap\left(Q_{11}-\tilde{Q}_{11}\right)=\emptyset$ and $\mathscr{S}(X)-Q_{11}$ contains only those $z$ for which (28) or (29) holds, i.e., $\mathscr{S}(X)=\mathscr{S}$.

The simple proof of Lemma A.1 is omitted.

Proof of Lemma A.2: We will prove the following statement, which directly implies Lemma A.2. 
Let $\tilde{A} \in S^{\tilde{p} \times \tilde{r}}, \tilde{B} \in S^{\tilde{p} \times \tilde{n}}$, and $\tilde{C} \in S^{\tilde{m} \times \bar{r}}$ be such that the smallest invariant factor (sif) of $\left[\begin{array}{cc}\tilde{A} & \tilde{B}\end{array}\right]$ and the sif of $\left[\begin{array}{ll}\tilde{A}^{\prime} & \tilde{C}^{\prime}\end{array}\right]^{\prime}$ are units, and either $\operatorname{rank} \bar{B} \geq 2$ or $\operatorname{rank} \tilde{C} \geq 2$. Then, for almost all $X \in S^{\tilde{n} \times \tilde{m}}, \operatorname{sif}(\tilde{A}+\tilde{B} X \tilde{C})$ is unit.

Proof: We can assume neither $\tilde{B}$ nor $\tilde{C}$ equals zero, because otherwise sif $\tilde{A}$ is unit, and the lemma holds trivially. If $X_{0}$ is such that $\operatorname{sif}\left(\tilde{A}+\tilde{B} X_{0} \tilde{C}\right)$ is unit, by choosing the norm of $\Delta$ small enough, $\operatorname{sif}\left(\tilde{A}+\tilde{B}\left(X_{0}+\right.\right.$ $\Delta) \tilde{C}$ ) is still a unit, since the set of units are open in $S$. To show that the class of such $X$ is dense, assume $X_{0}$ is such that $\operatorname{sif}\left(\tilde{A}+\tilde{B} X_{0} \tilde{C}\right)$ is not a unit of $S$. Let $U_{l}$ and $U_{r}$ be unimodular matrices of suitable size, such that

$$
U_{l} \tilde{B}=\left[\begin{array}{c}
\hat{B} \\
0
\end{array}\right], \text { and } \tilde{C} U_{r}=\left[\begin{array}{ll}
\hat{C} & 0
\end{array}\right]
$$

where $\hat{B} \in S^{\hat{p} \times \bar{n}}$ and full-row rank, and $\hat{C} \in S^{\bar{m} \times \hat{r}}$ and full-column rank. By assumption, either rank $\tilde{B} \geq 2$ or rank $\tilde{C} \geq 2$. We assume $\operatorname{rank} \tilde{B} \geq 2$. Otherwise rank $\tilde{C} \geq$ 2 and the dual of the proof below follows. Clearly, rank $\hat{B}$ $\geq 2$ implies $\hat{p} \geq 2$. Let $b$ and $c$ be the smallest invariant factors of $\hat{B}$ and $\hat{C}$, respectively. Define $B_{l}=\hat{B} / b$ and $C_{r}=\hat{C} / c$. There exist unimodular matrices $V_{l}$ and $V_{r}$ such that $V_{l} B_{l}=B, C_{r} V_{r}=C$, where the first row of $B$ is left unimodular and the first column of $C$ is right unimodular. Further define

$$
\bar{A}=\left[\begin{array}{cc}
V_{l} & 0 \\
0 & I
\end{array}\right] U_{l} \tilde{A} U_{r}\left[\begin{array}{cc}
V_{r} & 0 \\
0 & I
\end{array}\right] .
$$

Partition $\bar{A}$ as follows:

$$
\bar{A}=\left[\begin{array}{ll}
\overline{A_{11}} & \overline{A_{12}} \\
\overline{A_{21}} & \overline{A_{22}}
\end{array}\right]
$$

where $\overline{A_{11}} \in \boldsymbol{S}^{\hat{p} \times \hat{r}}, \overline{A_{12}} \in \boldsymbol{S}_{\tilde{p} \times \bar{r}-\hat{r}}, \overline{A_{21}} \in \boldsymbol{S}_{\tilde{p}}^{\bar{p}-\hat{p} \times \hat{r}}$, and $\overline{A_{22}}$ $\in S^{\tilde{p}-\hat{p} \times \tilde{r}-\hat{r}}$. Clearly, $\operatorname{sif}\left(\tilde{A}+\tilde{B}\left(X_{0}+\Delta\right) \tilde{C}\right)$ equals the sif of

$$
\bar{A}+b c\left[\begin{array}{l}
B \\
0
\end{array}\right]\left(X_{0}+\Delta\right)\left[\begin{array}{ll}
C & 0
\end{array}\right]
$$

for any $\Delta \in S^{\tilde{n} \times \tilde{m}}$. Define $A=\bar{A}_{11}+b c B X_{0} C$. Let us assume, without loss of generality that the first column of $A$ is nonzero, because otherwise there exists a perturbation $\Delta_{1}$ on $X_{0}$ with arbitrarily small norm such that the first column of $A$ in nonzero with $X_{0}$ is replaced by $X_{0}+\Delta_{1}$ (This is guaranteed by the fact that $B$ and the first column of $C$ are nonzero.) Also note that for any $z \in \mathscr{C}_{+e},(b c)=0$ and $\bar{A}_{11}=0$ imply

$$
\left[\begin{array}{cc}
0 & \overline{A_{12}} \\
\overline{A_{21}} & \overline{A_{22}}
\end{array}\right] \neq 0
$$

because of the hypothesis that $\operatorname{sif}\left[\begin{array}{ll}\tilde{A} & \bar{B}\end{array}\right]$ and $\operatorname{sif}\left[\tilde{A}^{\prime}\right.$ $\left.\tilde{C}^{\prime}\right]^{\prime}$ are units. Let $\sum_{i=1}^{\bar{n}} \beta_{i} b_{1 i}=1$, and $\sum_{i=1}^{\bar{m}} c_{i 1} \alpha_{i}=1$, for some $\alpha_{i}, i=1, \cdots, \tilde{m}$ and $\beta_{i}, i=1, \cdots, \tilde{n}$, where $b_{1 i}, i=$ $1, \cdots, \tilde{n}$ denote the first-row elements of $B$, and $c_{i 1}, i=$ $1, \cdots, \tilde{m}$ denote the first-column elements of $C$. Define
$\theta_{j}=\Sigma_{i=1}^{\bar{n}} \beta_{i} b_{j i}, j=1, \cdots, \hat{p}$, and $\gamma_{j}=\Sigma_{i=1}^{\bar{m}} c_{i j} \alpha_{i}, j=1, \cdots, \hat{r}$ where $b_{j i}$ denotes the $(j, i)$ th element of $B$ and $c_{i j}$ denotes the $(i, j)$ th element of $C$. Note that, $\theta_{1}=1$ and $\gamma_{1}=1$. By the fact that $\hat{p} \geq 2, \gamma_{1}=1$ and the first column of $A$ is nonzero, we can assume that for at least one index pair $(i, j), a_{i j} \neq \theta_{i} \gamma_{j} a_{11}$. (We omit the simple proof of the construction of such $\beta_{i}$ and $\alpha_{j}, i=1, \cdots, \tilde{n}$, and $j=1, \cdots, \tilde{m}$.) Now let $\Sigma$ be a nonempty set of index pairs so that $\Sigma=\left\{\left(i_{1}, j_{1}\right),\left(i_{2}, j_{2}\right), \cdots,\left(i_{v}, j_{v}\right)\right\}$ where $v=$ $\min (\hat{p}, \hat{r})$, satisfying $a_{i j} \neq \theta_{i} \gamma_{j} a_{11}$, whenever $(i, j) \in \Sigma$, and $a_{i j}=\theta_{i} \gamma_{j} a_{11}$, whenever $(i, j) \notin \Sigma$. Define $q_{i j}=$ $\operatorname{gcf}\left(a_{i j}, b c\right), i=1, \cdots, \hat{p}, j=1, \cdots, \hat{r}$, such that $a_{i j}=q_{i j} \hat{a}_{i j}$ and $b c=q_{i j} \hat{q}_{i j}$, for coprime pairs $\left(\hat{a}_{i j}, \hat{q}_{i j}\right)$. If $a_{11}=0$, let $\delta$ satisfy $\left(\delta, a_{i j}\right)$ are coprime for all $(i, j) \in \Sigma$. If $a_{11} \neq 0$, let $\delta$ satisfy $\left(\hat{a}_{11}+\delta \hat{q}_{11}, a_{i j}-\theta_{i} \gamma_{j} a_{11}\right)$ are coprime for all $(i, j) \in \Sigma$. The norm of $\delta$ can be chosen arbitrarily small in both cases. By letting

$$
\Delta=\left[\begin{array}{ccc}
\beta_{1} \delta \alpha_{1} & \cdots & \beta_{1} \delta \alpha_{\bar{m}} \\
\vdots & & \vdots \\
\beta_{\tilde{n}} \delta \alpha_{1} & \cdots & \beta_{\tilde{n}} \delta \alpha_{\bar{m}}
\end{array}\right]
$$

we have $(A+b c B \Delta C)_{i j}=a_{i j}+\delta b c \theta_{i} \gamma_{j}, i=1, \cdots, \hat{p}, j=$ $1, \cdots, \hat{r}$. If $a_{11}=0$, the choice of $\Delta$ yields

$$
g c f_{i=1, \cdots, \hat{p}, j=1, \ldots, \hat{r}}\left[(A+b c B \Delta C)_{i j}\right]=g c f_{(i, j) \in \Sigma}\left(a_{i j}, b c\right) .
$$

In the case that $a_{11} \neq 0$, the choice of $\Delta$ yields

$$
g c f_{i=1 ;,-\hat{p}, j=1,-\hat{r}}\left[(A+b c B \Delta C)_{i j}\right]=g c f_{(i, j) \in \Sigma}\left(a_{i j}, q_{11}\right) \text {. }
$$

(This latter statement can be seen more clearly as follows. Observe that whenever $(i, j) \notin / \Sigma, g c f\left[(A+B \Delta C b c)_{11}\right.$, $\left.(A+B \Delta C b c)_{i j}\right]=a_{11}+b c \delta$. So

$g c f_{i=1,-, \hat{p}, j=1, ;, \hat{r}}\left[(A+b c B \Delta C)_{i j}\right]$

$$
=g c f\left(a_{11}+b c \delta, g c f_{(i, j) \in \Sigma}(A+b c B \Delta C)_{i j}\right) .
$$

$\left(\hat{a}_{11}+\delta \hat{q}_{11}\right)=0, z \in \mathscr{C}_{+e}$ imply $\delta(z)=-\hat{a}_{11} / \hat{q}_{11}$, where $\hat{q}_{11}$ is nonzero because of the coprimeness of $\left(\hat{a}_{11}, \hat{q}_{11}\right)$. In this case $\left(a_{i j}+b c \delta \theta_{i} \gamma_{j}\right)=\left(a_{i j}-\theta_{i} \gamma_{j} a_{11}\right) \neq 0$. Hence

$$
\begin{aligned}
g c f( & \left.a_{11}+b c \delta, g c f_{(i, j) \in \Sigma}(A+B \Delta C b c)_{i j}\right) \\
& =g c f\left(q_{11}, g c f_{(i, j) \in \Sigma}(A+B \Delta C b c)_{i j}\right) \\
& \left.=g c f_{(i, j) \in \Sigma}\left(q_{11}, a_{i j}\right) .\right)
\end{aligned}
$$

In both cases $\operatorname{sif}(A+B \Delta C b c)$ is coprime with

$$
\text { sif }\left[\begin{array}{cc}
0 & \bar{A}_{12} \\
\bar{A}_{21} & \overline{A_{22}}
\end{array}\right] \text {. }
$$

Since the norm of $\Delta$ can be made smaller than any prespecified positive number by choosing $\delta$ suitably, the proof is completed.

\section{REFERENCES}

[1] C.-C. Chu and F.-R. Chang, "Some results on the problems of decentralized reliable stabilization," Int. J. Contr., vol. 53, no. 6, pp. 1343-1358, 1991.

[2] J. P. Corfmat and A. S. Morse, "Decentralized control of linear multivariable systems," Automatica, vol. 8, pp. 479-495, 1976. 
[3] N. Gündeş and C. A. Desoer, Algebraic Theory of Linear Feedback Systems with Full Decentralized Compensators. Berlin: SpringerVerlag, 1990.

[4] P. P. Khargonekar, K. Poolla, and A. Tannenbaum, "Robust control of linear time-invariant plants using periodic compensators," IEEE Trans. Automat. Contr., vol. AC-30, no. 11, pp. 1088-1096, 1985.

[5] X. Minto and X. Ravi, "New results on the multi-controller scheme for the reliable control of linear plants," in Proc. $A C C, 1991$, pp. $615-619$.

[6] Y. Ohta, D. D. Šiljak, and T. Matsumoto, "Decentralized control using quasi-block diagonal dominance of transfer function matrices," IEEE Trans. Automat. Contr., vol. AC-31, pp. 420-429, 1986.

[7] A. B. Özgüler, "Decentralized control: A stable proper fractional approach," IEEE Trans. on Automat. Control, vol. 35, no. 10, pp. $1109-1117,1990$.

[8] A. B. Özgüler and M. Hiraoğlu, "Implications of a characterization result on strong and reliable decentralized control," in Modelling, Robustness and Sensitivity Reduction in Control Systems (NATO ASI Series, vol. F34), R. F. Curtain, Ed. 1987.

[9] M. E. Sezer and Ö. Hüseyin, "Stabilization of linear time-invariant interconnected systems using local state feedback," IEEE Trans. Syst. Man. Cybern., vol. SMC-8, pp. 751-756, 1978.

[10] D. D. Siljak, "Reliable control using multiple control systems," Int. J. Contr., vol. 31, pp. 303-329, 1980.

[11] X.-L. Tan and M. Ikeda, "Decentralized stabilization for expanding construction of large-scale systems," IEEE Trans. Automat. Contr., vol. 35, pp. 644-651, 1990.

[12] K. A. Ünyelioğlu, and A. B. Özgüler, "Decentralized stabilization of multivariable systems using a stable proper fractional approach," in Proc. 1990 Bilkent Int. Conf. Commun., Contr. Signal Proc., 1990, vol. 1, pp. 843-849.

[13] K. A. Ünyelioğlu and A. B. Özgüler, "Decentralized strong stabilization problem," in Proc. Amer. Contr. Conf., 1992.

[14] R. J. Veillette, J. V. Medanić, and W. R. Perkins, "Design of reliable control systems," in Proc. 29th Conf. Decision Contr., Honolulu, HI, 1990, pp. 1131-1136.

[15] M. Vidyasagar, Control System Synthesis: A Factorization Approach. Cambridge, MA: M.I.T. Press, 1985.

[16] M. Vidyasagar and N. Viswanadham, "Reliable stabilization using a multi-controller configuration," Automatica, vol. 21, pp. 599-602, 1985 .

[17] S. H. Wang and E. J. Davison, "On the stabilization of decentralized control systems," IEEE Trans. Automat. Contr., vol. 18, pp. $473-478,1974$.
[18] Q.-H. Wu and M. Mansour, "Decentralized robust control using $H^{\star}$-optimization technique," in Proc. 27th Conf. Decision Contr., Austin, TX, 1988, pp. 1335-1340.

[19] D. C. Youla, J. J. Bongiorno, Jr., and C. N. Lu, "Single-loop feedback stabilization of linear multivariable plants," Automatica, vol. 10, pp. 159-173, 1974.

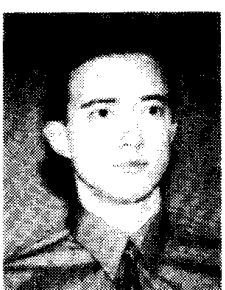

Konur Alp Ünyelioğlu (S'89--M'91) was born in Turkey in 1964. He received the B.S. degree from the Middle East Technical University,

Ankara, Turkey, and the M.S. degree from Bilkent University, Ankara, Turkey, in 1986 and 1988 , respectively, all in electrical and electronics engineering.

He was with the University of Michigan, Ann Arbor, during the fall semester of 1991 as a Visiting Scholar. He is currently a Ph.D. student in the Department of Electrical and Electronics Engineering, Bilkent University, Ankara, Turkey. His research interests include decentralized stabilization problems for linear time-invariant and time-varying sytems and infinite dimensional systems using statespace and factorization methods.

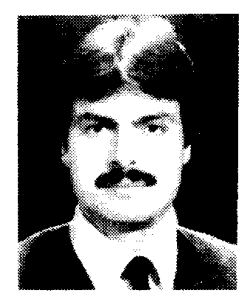

A. Bülent Özgüler was born in Gaziantep, Turkey, on December 18,1954 . He received the B.Sc. and M.Sc. degrees from the Middle East Technical University, Ankara, Turkey, in 1976 and 1978, respectively, and the Ph.D. degree from the Center for Mathematical System The ory, University of Florida, Gainesville, in 1982 all in electrical engineering.

He was a Researcher at the Applied Mathematics Division of Marmara Research Institute, Kocaeli, Turkey, from 1983 to 1986 . He is currently with the Department of Electrical and Electronics Engineering, Bilkent University, Ankara, Turkey. His major research interests are in the field of mathematical control theory.

Dr. Özgüler is a recipient of the 1985 Sedat Simavi Foundation Award for applied sciences and of a research prize of the Scientific and Technological Research Council of Turkey (TÜBITAK) in 1987. 